\title{
A survey of molecular line emission towards ultracompact HII regions $^{\star, \star \star}$
}

\author{
J. Hatchell ${ }^{1}$, M.A. Thompson ${ }^{2}$, T.J. Millar ${ }^{1}$, and G.H. Macdonald ${ }^{2}$ \\ 1 Department of Physics, UMIST, P.O. Box 88, Manchester M60 1QD, UK \\ 2 Electronic Engineering Laboratory, University of Kent, Canterbury, Kent CT2 7NT, UK
}

Received August 18, 1997; accepted May 13, 1998

\begin{abstract}
We have used the JCMT to survey molecular line emission towards 14 ultracompact HiI regions (G5.89, G9.62, G10.30, G10.47, G12.21, G13.87, G29.96, G31.41, G34.26, G43.89, G45.12, G45.45, G45.47, and G75.78). For each source, we observed up to ten $1 \mathrm{GHz}$ bands between 200 and $350 \mathrm{GHz}$, covering lines of more than 30 species including multiple transitions of $\mathrm{CO}$ isotopes, $\mathrm{CH}_{3} \mathrm{OH}, \mathrm{CH}_{3} \mathrm{CCH}, \mathrm{CH}_{3} \mathrm{CN}$ and $\mathrm{HCOOCH}_{3}$, and sulphuretted molecules. The number of transitions detected varied by a factor of 20 between sources, which were chosen following observations of high-excitation ammonia (Cesaroni et al. 1994a) and methyl cyanide (Olmi et al. 1993). In half our sample (the line-poor sources), only $\mathrm{C}^{17} \mathrm{O}, \mathrm{C}^{18} \mathrm{O}$, SO, $\mathrm{C}^{34} \mathrm{~S}$ and $\mathrm{CH}_{3} \mathrm{OH}$ were detected. In the line-rich sources, we detected over 150 lines, including high excitation lines of $\mathrm{CH}_{3} \mathrm{CN}, \mathrm{HCOOCH}_{3}, \mathrm{C}_{2} \mathrm{H}_{5} \mathrm{CN}$, $\mathrm{CH}_{3} \mathrm{OH}$, and $\mathrm{CH}_{3} \mathrm{CCH}$. We have calculated the physical conditions of the molecular gas. To reproduce the emission from the line-rich sources requires both a hot, dense compact core and an ambient cloud consisting of less dense, cooler gas. The hot cores, which are less than $0.1 \mathrm{pc}$ in size, reach densities of at least $10^{8} \mathrm{~cm}^{-3}$ and temperatures of more than $80 \mathrm{~K}$. The line-poor sources can be modelled without a hot core by a $20-30 \mathrm{~K}, 10^{5} \mathrm{~cm}^{-3}$ cloud. We find no correlation between the size of the Hir region and the current physical conditions in the molecular environment. A comparison with chemical models (Millar et al. 1997) confirms that grain surface chemistry is important in hot cores.
\end{abstract}

Key words: ISM: molecules - ISM: clouds — ISM: HII regions - radio lines: ISM — stars: formation molecular processes

\footnotetext{
Send offprint requests to: J. Hatchell

* Tables 10-18 are only available in electronic form at the CDS via anonymous ftp to cdsarc.u-strasbg.fr (130.79.128.5) or via http://cdsweb.u-strasbg.fr/Abstract.html

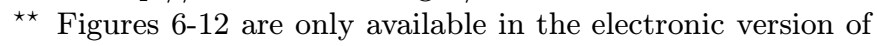
the journal at http://www.edpsciences.com
}

\section{Introduction}

In many regions of recent massive star formation, indicated by the presence of ultracompact (UC) HiI regions, there are hot, dense cores of molecular gas. These cores are characterised physically by their small volumes, high temperatures and high densities and chemically by high abundances of saturated molecules. The molecular gas lies close to the UC Hil region and may be heated externally or by embedded young stars. Hot cores are the natal molecular clouds for high mass stars and by understanding their physical and chemical properties we hope to shed light on the process of high mass star formation.

Hot cores provide a unique opportunity to investigate the composition of grain mantles via observations of species in the gas phase. As the density increases during collapse to form a core, molecules freeze out onto dust grains and then undergo processing on the grains. As the cores heat, temperatures become high enough to evaporate molecules from ices, injecting them into the gas phase and leading to a rich chemistry. High abundances of saturated molecules are believed to be a hallmark of grain mantle evaporation as species are hydrogenated on grains. Unlike gas phase chemistry, the chemistry that occurs in the grain ices is poorly understood. Molecules are difficult to detect and distinguish in the solid ice phase, and any species with an abundance less than $1 \%$ of solid $\mathrm{H}_{2} \mathrm{O}$ is essentially undetectable. In hot cores in the gas phase, densities are high enough for large numbers of species to be detected, and we are able to constrain the composition of the ice mantles by looking at molecular abundances of the evaporated molecules from grains.

Another motivation for a survey of molecular gas associated with HiI regions is to look at the effects of the environment on the morphology and size of the ionised region. The molecular cores in our sample were discovered by Churchwell et al. (1990) while investigating the environment of UC HII regions. UC HII regions come with several different types of morphology, classified as cometary (e.g. G34.26, G29.96), core-halo (G31.41), spherical or 
unresolved (G10.47), shell (G5.89) and irregular (G45.45). A unifying explanation for the expansion of UC HII regions, which appear smaller than one would naïvely expect from their lifetimes, and the creation of these different morphologies, has not been settled on. In our molecular line survey we obtain measures of the density and the kinematics of the molecular environment of the HıI region. In Sect. 4.2 we compare these to requirements and predictions of the HII expansion models.

Whether the hot cores are heated solely by interaction with the neighbouring HII regions, or internally by secondary star formation, their chemical composition will reflect their history. Molecules released from the grain ices are processed in the gas phase, changing their abundance ratios as time progresses. Differences in abundance ratios between sources may be evidence of evolution. By comparison with chemical models, such as the model for G34.26 (Millar et al. 1997) we can estimate the ages of the hot cores. This approach has been successfully used to rank three sites of high mass star formation in W3 in order of age (Helmich et al. 1994). We consider the chemical evidence for evolutionary differences between the hot cores in our survey in a subsequent paper (Hatchell et al. 1998, in preparation).

Our sample of fourteen hot cores (see Table 1) was chosen to consist of cores known to contain hot gas because they have been observed in emission from high excitation lines of ammonia $((4,4)$ and $(5,5)$, Cesaroni et al. 1992) and methyl cyanide (Olmi et al. 1993). The sample also includes the archetypal hot core source G34.26. We have previously published an extended molecular line survey of G34.26 and compared it with a detailed chemical model (Macdonald et al. 1996; Millar et al. 1997). Subsamples of our selection have been observed in the dense gas tracers $\mathrm{CS}$ and $\mathrm{C}^{34} \mathrm{~S}$ (Cesaroni et al. 1991; Plume et al. 1992; Churchwell et al. 1992). Enhanced abundances of deuterium have been observed in G34.26, G10.47, G31.41, G29.96 and G9.62, supporting the argument that material is being evaporated from ices as deuterated molecules form preferentially in cold conditions (Jacq et al. 1990; Gensheimer et al. 1996; Rodgers \& Millar 1996). Most of the hot core/UC HiI complexes have associated $\mathrm{H}_{2} \mathrm{O}$ masers, which are strongly associated with recent star formation (Churchwell et al. 1990; Hofner \& Churchwell 1996; Codella et al. 1997), and some (if not all) have outflow activity (Cesaroni et al. 1991; Shepherd \& Churchwell 1996; Hunter et al. 1997; Olmi et al. 1996a; Hofner et al. 1996; Shepherd et al. 1997).

We choose frequency bands that include transitions of many different molecules. We include two sets of high excitation transitions of $\mathrm{CH}_{3} \mathrm{CN}$ (methyl cyanide) as a temperature tracer in hot, dense gas. $\mathrm{CH}_{3} \mathrm{CN}$ is a symmetric top molecule with groups of transitions closely spaced in frequency but involving widely spaced energy levels. It is used as a temperature probe because the lines within a group can be observed together, thus removing the need for various calibration factors to be taken into account when comparing line ratios. We include many lines of $\mathrm{CH}_{3} \mathrm{OH}$ (methanol) which span a large range of excitation energies and trace both hot and cold gas. $\mathrm{C}^{17} \mathrm{O}$ and $\mathrm{C}^{18} \mathrm{O}$ are observed as tracers of column density. Other molecules with multiple transitions which can be used for temperature and density analysis are $\mathrm{CH}_{3} \mathrm{CCH}$ (methyl acetylene) and $\mathrm{HCOOCH}_{3}$ (methyl formate). Observations include a number of sulphur species which are good tracers of chemical evolution.

Following a description of the observations made (Sect. 2), we present the results of the analysis moleculeby-molecule (Sect. 3.2), first briefly describing the techniques used. In Sect. 4 we draw together the results from individual species to make general statements about the physical conditions and structure, the implications for UC HII models, and the chemistry and evolution of the molecular gas. Finally, Sect. 5 summarises our conclusions.

\section{Observations}

Observations of all molecular lines in this survey were made using the James Clerk Maxwell Telescope $\left(\right.$ JCMT) ${ }^{1}$ in September 1995 and April 1996. We used the commonuser receivers A2 (230 GHz band) and B3i (345 GHz band) coupled to the DAS (Dutch Autocorrelation Spectrometer). The bandwidth was a nominal $760 \mathrm{MHz}$, giving a frequency resolution of $0.756 \mathrm{MHz}$. Each spectrum was divided into channels of width $0.625 \mathrm{MHz}$. In September 1995, we tuned to the frequencies of lines of $\mathrm{H}_{2} \mathrm{~S} 2(2,0)-2(2,1)(216.710 \mathrm{GHz}), \mathrm{CH}_{3} \mathrm{CN} J=13-12$ $K=0(239.138 \mathrm{GHz}), \mathrm{C}^{17} \mathrm{O} J=3-2(337.061 \mathrm{GHz})$ and $\mathrm{CH}_{3} \mathrm{CN} J=19-18(349.350 \mathrm{GHz})$. All data were taken in dual sideband mode, with sidebands centred $\pm 3 \mathrm{GHz}$ from the main band. Counting both main and image (upper or lower) sidebands, we observed between two and ten $760 \mathrm{MHz}$-wide bands in each source. Pointing was checked on G34.26 and was accurate to within $5^{\prime \prime}$ throughout the observations. System temperatures were in the range $400-650 \mathrm{~K}$ with $\mathrm{RxA} 2$ and $900-1300 \mathrm{~K}$ with RxB3i.

Additional spectra of $\mathrm{C}^{17} \mathrm{O} J=2-1(224.714 \mathrm{GHz})$ and $\mathrm{C}^{18} \mathrm{O} J=3-2(329.331 \mathrm{GHz})$ were taken in April 1996. System temperatures were higher at $600-700 \mathrm{~K}$ with $\mathrm{RxA}$ and $4500-10000 \mathrm{~K}$ with $\mathrm{RxB}$. The resulting rms noise at $329 \mathrm{GHz}$ was between 0.4 and $0.9 \mathrm{~K}$, and $0.05 \mathrm{~K}$ at $225 \mathrm{GHz}$.

The spectra of G5.89 were obtained during March 1996 as part of a $300-360 \mathrm{GHz}$ molecular line survey of this source (Thompson \& Macdonald 1998, in preparation).

1 The James Clerk Maxwell Telescope is operated by The Joint Astronomy Centre on behalf of the Particle Physics and Astronomy Research Council of the United Kingdom, the Netherlands Organisation for Scientific Research, and the National Research Council of Canada. 
Table 1. Positions, velocities and distances for observed objects

\begin{tabular}{|c|c|c|c|c|c|c|c|c|}
\hline \multirow[t]{2}{*}{$\overline{\text { Object }}$} & \multicolumn{3}{|c|}{$\alpha_{1950}$} & \multicolumn{3}{|c|}{$\delta_{1950}$} & \multirow{2}{*}{$\begin{array}{c}v_{\mathrm{LSR}} \\
{\left[\mathrm{km} \mathrm{s}^{-1}\right.}\end{array}$} & \multirow{2}{*}{$\begin{array}{c}d \\
{[\mathrm{kpc}}\end{array}$} \\
\hline & {$[\mathrm{h}$} & & $\mathrm{s}$ & & & " & & \\
\hline G5.89-0.39 & 17 & 57 & 26.8 & -24 & 03 & 56 & 9.0 & 2.5 \\
\hline G9.62+ & 18 & 03 & 16.2 & -20 & 32 & 03 & 4.4 & 5.7 \\
\hline G10.30-0.15 & 18 & 05 & 57.9 & -20 & 06 & 26 & 13. & 6.0 \\
\hline G10.47+0.03 & 18 & 05 & 40.3 & -19 & 52 & 21 & 67.8 & 5.8 \\
\hline G12.21-0.10 & 18 & 09 & 43.7 & -18 & 25 & 09 & 24.0 & 13.5 \\
\hline $\mathrm{G} 13.87+0.28$ & 18 & 11 & 41.8 & -16 & 46 & 40 & 48. & 4.4 \\
\hline G29. & 18 & 43 & 27.1 & -02 & 42 & 36 & 97.6 & 7.4 \\
\hline G31.41+0.31 & 18 & 44 & 59.4 & -01 & 16 & 04 & 97.4 & 7.9 \\
\hline G34.26+0.15 & 18 & 50 & 46.1 & 01 & 11 & 12 & 58.0 & 4.0 \\
\hline G43.89-0.78 & 19 & 12 & 02.8 & 09 & 17 & 19 & 53.4 & 4.2 \\
\hline $\mathrm{G} 45.12+0.13$ & 19 & 11 & 06.2 & 10 & 48 & 26 & 58.6 & 6.9 \\
\hline $\mathrm{G} 45.45+0.06$ & 19 & 12 & 00.1 & 11 & 03 & 60 & 57.0 & 6.6 \\
\hline $\mathrm{G} 45.47+0.05$ & 19 & 12 & 04.4 & 11 & 04 & 11 & 62.0 & 6.0 \\
\hline G75.78+0.34 & 20 & 19 & 52.0 & 37 & 17 & 02 & -0.1 & 4.1 \\
\hline
\end{tabular}

These are by far the noisiest spectra with rms noises of between 0.08 and $0.18 \mathrm{~K}$ in $0.625 \mathrm{MHz}$ channels.

With many lines visible in each spectrum, it can be difficult to attribute lines to main and image sidebands. In some cases we changed the local oscillator frequency by $10 \mathrm{MHz}$ and observed a second spectrum in which the image sideband lines appeared shifted. In many cases we observed with lower and then upper sideband centred on the tuning frequency, so that different lines appeared in the image sideband. Line identification was performed with the help of laboratory and theoretical lists of millimetre transitions (Lovas 1985; Poynter \& Pickett 1985; Anderson et al. 1990a,b; Boucher et al. 1980, Herbst 1996) All spectra and line temperatures quoted in this paper have been corrected for telescope forward scattering and spillover efficiency $\eta_{\text {fss }}$ to give the main beam brightness temperature $T_{\mathrm{R}}^{*}$ in kelvin appropriate for extended sources. $\eta_{\mathrm{fss}}$ was taken to be 0.8 in the $230 \mathrm{GHz}$ band and 0.7 in the $345 \mathrm{GHz}$ band. Although the hot cores are smaller than the JCMT beam (which is $\sim 21^{\prime \prime}$ in the RxA band and $\sim 14^{\prime \prime}$ with $\mathrm{RxB}$ ) we are also observing ambient cloud emission on larger angular scales, hence our correction for $\eta_{\mathrm{fss}}$ as measured on the moon. The conversion from $T_{\mathrm{R}}^{*}$ to flux $S$ in $\mathrm{Jy}$ is given for the JCMT by $(S / \mathrm{Jy}) \simeq 13\left(T_{\mathrm{R}}^{*} / \mathrm{K}\right)$.

The observed sources are listed in Table 1 with the positions, distances (Churchwell et al. 1990) and LSR velocities (from ammonia; Cesaroni et al. 1992; Olmi et al. 1993) assumed. In Table 2, the frequency bands in which each object was observed are shown. Not all sources were observed in all frequency bands. In general, the sources which showed the most line emission were observed at more frequencies. Spectra were taken towards the HiI region positions in all sources. Spectra were also observed at an offset position $20^{\prime \prime}$ to the north of the HII region in five cores: G9.62, G10.47, G29.96, G31.41 and G34.26.

\section{Results and analysis}

Appendix A (in the electronic version of this paper only: see appendix for details) contains the molecular spectra observed towards each UC Hit region. Figure 1 shows examples from four sources. In Appendix B (available only in electronic form from CDS: see appendix for details) we list the line detections for each source by frequency, with identifications and line parameters. Hot core sources are some of the richest known sources in molecular line emission. In total, we have identified more than 150 molecular lines in some sources, in less than $10 \mathrm{GHz}$ total bandwidth. The variation between sources is great, with the number of molecular lines identified with reasonable signal-to-noise varying by a factor of 20 between chemically-rich and chemically-poor sources. Some sources show many highexcitation lines from complex molecules, whereas others show little emission. In G45.47, G10.30, G43.89 and G13.87, only a few molecular lines were detected: $\mathrm{C}^{17} \mathrm{O}$ and $\mathrm{C}^{18} \mathrm{O}, \mathrm{SO}, \mathrm{C}^{34} \mathrm{~S}$, and weak emission from the lowest excitation energy lines of $\mathrm{CH}_{3} \mathrm{CCH}$ and $\mathrm{CH}_{3} \mathrm{OH}$. G45.45 and G45.12 showed no emission lines in the $239 \mathrm{GHz}$ band, and were not observed at other frequencies. We label these "line-poor" sources. In contrast, the sources G34.26, G10.47 and G31.41 show emission from high excitation energy lines of $\mathrm{CH}_{3} \mathrm{OH}, \mathrm{CH}_{3} \mathrm{CCH}$ and $\mathrm{CH}_{3} \mathrm{CN}$, and also lines of $\mathrm{C}_{2} \mathrm{H}_{5} \mathrm{CN}, \mathrm{CH}_{2} \mathrm{CHCN}, \mathrm{CH}_{3} \mathrm{CHO}, \mathrm{CH}_{3}^{13} \mathrm{CN},{ }^{13} \mathrm{CH}_{3} \mathrm{OH}$, $\left(\mathrm{CH}_{3}\right)_{2} \mathrm{O}, \mathrm{H}_{2}^{13} \mathrm{CO}, \mathrm{HCOOCH}_{3}, \mathrm{SiS}$, and $\mathrm{SO}_{2}$. Five further sources, G5.89, G9.62, G12.21, G29.96 and G75.78, are also line-rich, showing a number of high excitation lines. The $20^{\prime \prime}$ offset positions observed in G9.62, G10.47, G29.96, G31.41 and G34.26 show only a small number of lines, with spectra similar to the line-poor sources.

Below, we describe the techniques used in the analysis of the molecular line data and then examine the line excitation molecule-by-molecule.

\subsection{Analysis}

We have studied the excitation of the hot cores using a number of techniques, and some quantitative methods are reused for several different molecules. To avoid repetition, we briefly outline these techniques here. The results of the excitation studies for individual molecules are given below.

In order to make comparisons with chemical models, we are particularly interested in molecular column densities, as these translate into fractional abundances where the total hydrogen column density is known. For one or two detected transitions of a molecule, it is only possible to put a lower limit on the column density, making assumptions about the excitation conditions. Where multiple transitions involving different energy levels are observed for the same molecule, then it is possible to make a more detailed examination of the excitation. Molecules 


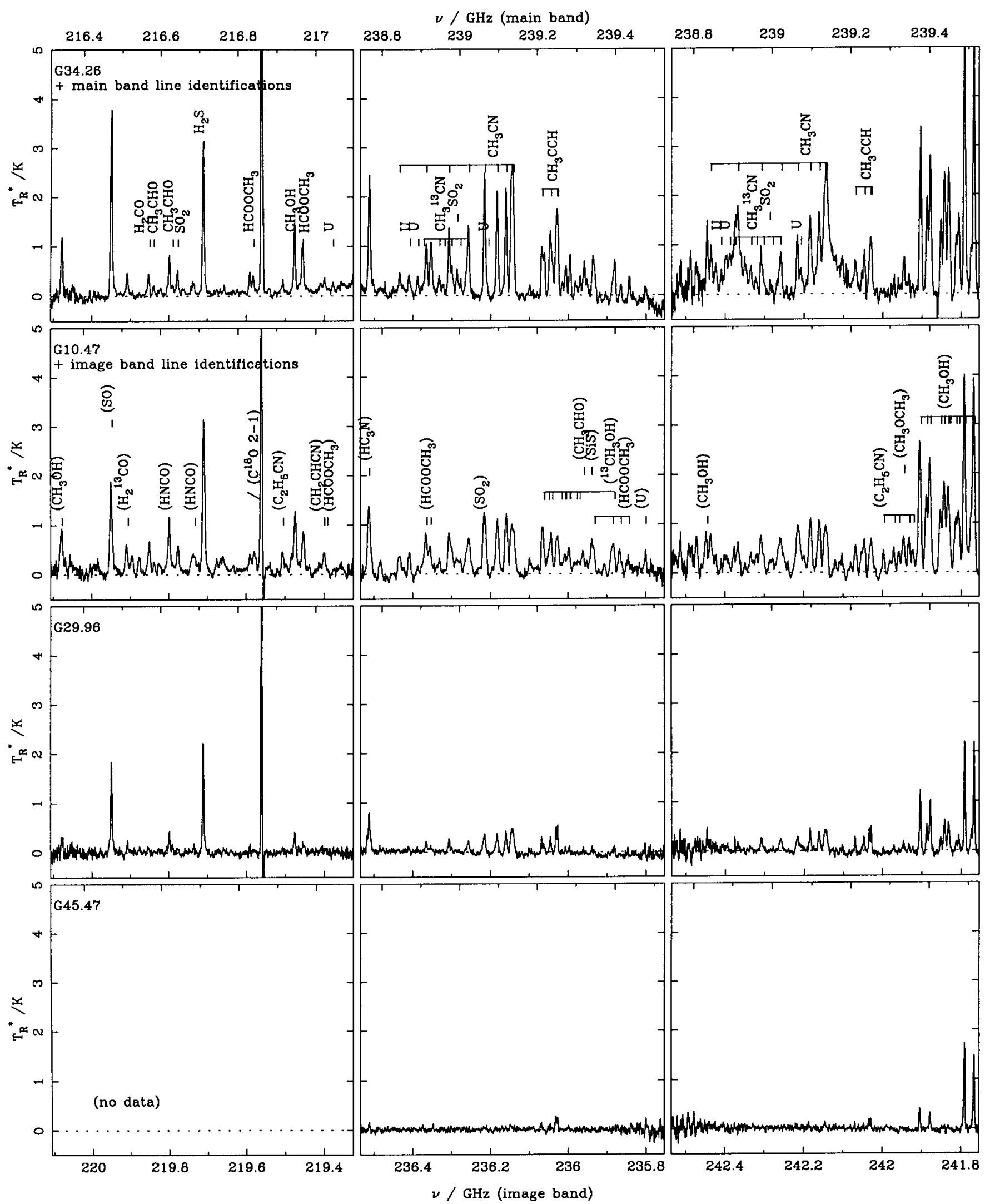

Fig. 1. Examples of spectra in the six main frequency bands for four sources (G34.26, G10.47, G29.96 and G45.47). Lines from the main band (upper frequency scale) are identified on the G34.26 spectra and those from the image band (lower frequency scale, identifications in parentheses) on the G10.47 spectra. Spectra for all the sources are available electronically (see Appendix A) 


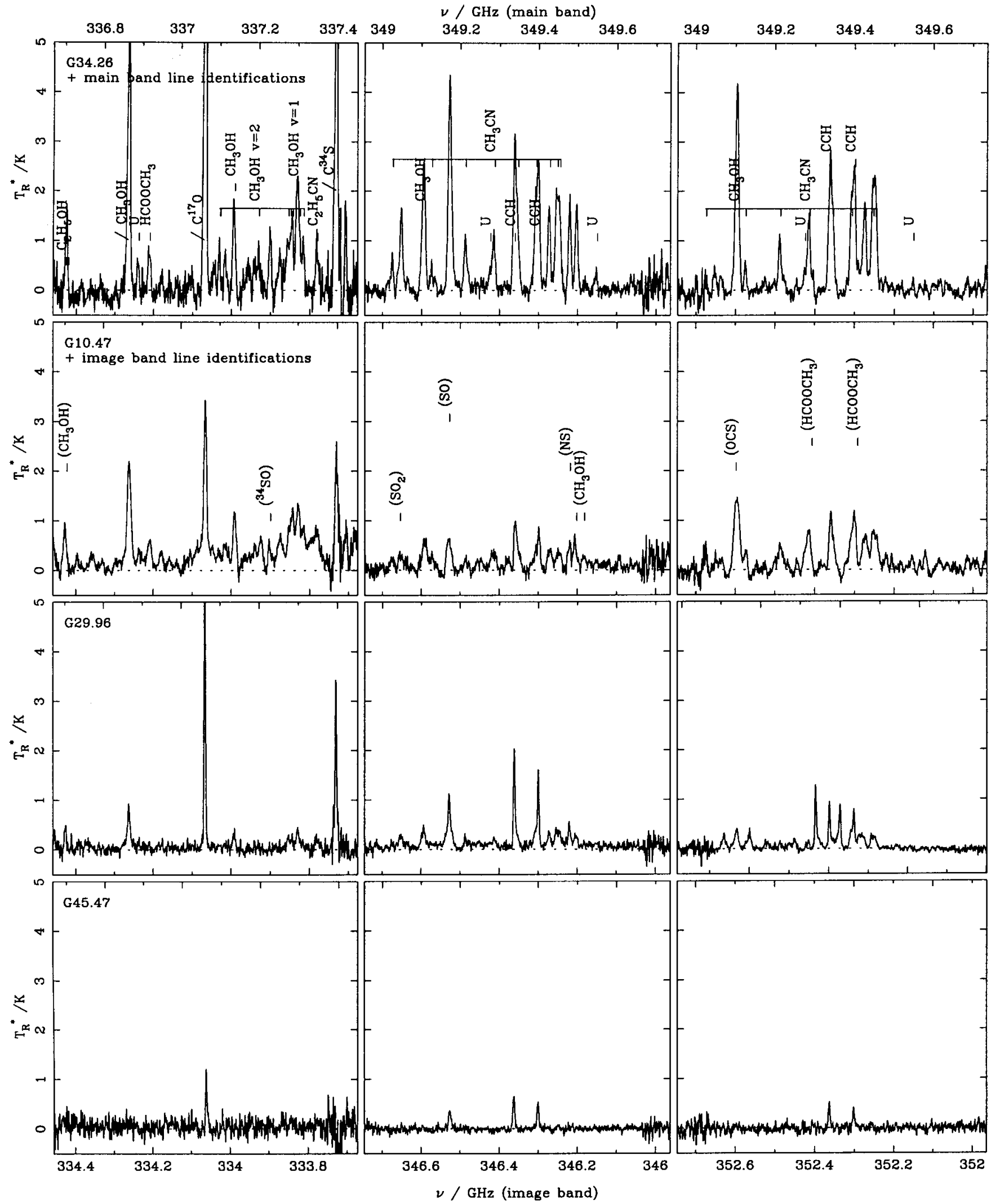

Fig. 1. continued 
Table 2. Observed frequency bands for each source, with important molecules with transitions in each band given

\begin{tabular}{|c|c|c|c|c|c|c|c|c|c|c|c|c|}
\hline$\nu / \mathrm{GHz}$ & $\begin{array}{c}216 \\
\mathrm{H}_{2} \mathrm{~S}\end{array}$ & $\begin{array}{c}219 \\
\mathrm{C}^{18} \mathrm{O}\end{array}$ & $\begin{array}{c}224 \\
\mathrm{C}^{17} \mathrm{O}\end{array}$ & $\begin{array}{c}236 \\
{ }^{13} \mathrm{CH}_{3} \mathrm{OH}\end{array}$ & $\begin{array}{c}239 \\
\mathrm{CH}_{3} \mathrm{CN}\end{array}$ & $\begin{array}{c}242 \\
\mathrm{CH}_{3} \mathrm{OH}\end{array}$ & $\begin{array}{c}329 \\
\mathrm{C}^{18} \mathrm{O}\end{array}$ & 334 & $\begin{array}{c}337 \\
\mathrm{C}^{17} \mathrm{O}\end{array}$ & $\begin{array}{l}346 \\
\text { SO }\end{array}$ & $\begin{array}{c}349 \\
\mathrm{CH}_{3} \mathrm{CN}\end{array}$ & 352 \\
\hline Source & $\mathrm{SO}_{2}$ & $\mathrm{SO}$ & & & $\mathrm{CH}_{3} \mathrm{CCH}$ & & & & $\mathrm{CH}_{3} \mathrm{OH}$ & $\mathrm{SO}_{2}$ & & \\
\hline G5.89-0.39 & & & & & & & $\mathrm{y}$ & & $\mathrm{y}$ & & $\mathrm{y}$ & $\mathrm{y}$ \\
\hline G9.62+0.19 & $\mathrm{y}$ & $\mathrm{y}$ & & $\mathrm{y}$ & $\mathrm{y}$ & $\mathrm{y}$ & $\mathrm{y}$ & $\mathrm{y}$ & $\mathrm{y}$ & $\mathrm{y}$ & $\mathrm{y}$ & $\mathrm{y}$ \\
\hline G10.30-0.15 & & & & $\mathrm{y}$ & $\mathrm{y}$ & $\mathrm{y}$ & & $\mathrm{y}$ & $\mathrm{y}$ & & & \\
\hline $\mathrm{G} 10.47+0.03$ & $\mathrm{y}$ & $\mathrm{y}$ & & $\mathrm{y}$ & $\mathrm{y}$ & $\mathrm{y}$ & & $\mathrm{y}$ & $\mathrm{y}$ & $\mathrm{y}$ & $\mathrm{y}$ & $\mathrm{y}$ \\
\hline G12.21-0.10 & & & & $\mathrm{y}$ & $\mathrm{y}$ & $\mathrm{y}$ & $\mathrm{y}$ & $\mathrm{y}$ & $\mathrm{y}$ & $\mathrm{y}$ & $\mathrm{y}$ & $\mathrm{y}$ \\
\hline $\mathrm{G} 13.87+0.28$ & & & & $\mathrm{y}$ & $\mathrm{y}$ & $\mathrm{y}$ & & $\mathrm{y}$ & $\mathrm{y}$ & & & \\
\hline G29.96-0.02 & $\mathrm{y}$ & $\mathrm{y}$ & & $\mathrm{y}$ & $\mathrm{y}$ & $\mathrm{y}$ & $\mathrm{y}$ & $\mathrm{y}$ & $\mathrm{y}$ & $\mathrm{y}$ & $\mathrm{y}$ & $\mathrm{y}$ \\
\hline G31.41+0.31 & $\mathrm{y}$ & $\mathrm{y}$ & $\mathrm{y}$ & $\mathrm{y}$ & $\mathrm{y}$ & $\mathrm{y}$ & $\mathrm{y}$ & $\mathrm{y}$ & $\mathrm{y}$ & $\mathrm{y}$ & $\mathrm{y}$ & $\mathrm{y}$ \\
\hline G34.26+0.15 & $\mathrm{y}$ & $\mathrm{y}$ & $\mathrm{y}$ & $\mathrm{y}$ & $\mathrm{y}$ & $\mathrm{y}$ & $\mathrm{y}$ & $\mathrm{y}$ & $\mathrm{y}$ & $\mathrm{y}$ & $\mathrm{y}$ & $\mathrm{y}$ \\
\hline G43.89-0.78 & $\mathrm{y}$ & $\mathrm{y}$ & & $\mathrm{y}$ & $\mathrm{y}$ & $\mathrm{y}$ & $\mathrm{y}$ & $\mathrm{y}$ & $\mathrm{y}$ & & & \\
\hline G45.12+0.13 & & & & & $\mathrm{y}$ & $\mathrm{y}$ & & & & & & \\
\hline G45.45+0.06 & & & & & $\mathrm{y}$ & $\mathrm{y}$ & & & & & & \\
\hline $\mathrm{G} 45.47+0.05$ & & & & $\mathrm{y}$ & $\mathrm{y}$ & $\mathrm{y}$ & & $\mathrm{y}$ & $\mathrm{y}$ & $\mathrm{y}$ & $\mathrm{y}$ & $\mathrm{y}$ \\
\hline G75.78+0.34 & $\mathrm{y}$ & $\mathrm{y}$ & & $\mathrm{y}$ & $\mathrm{y}$ & $\mathrm{y}$ & $\mathrm{y}$ & $\mathrm{y}$ & $\mathrm{y}$ & $\mathrm{y}$ & $\mathrm{y}$ & $\mathrm{y}$ \\
\hline
\end{tabular}

which have multiple transitions covering a wide range of excitation energies in a small frequency range, such as $\mathrm{CH}_{3} \mathrm{OH}, \mathrm{CH}_{3} \mathrm{CCH}$ and $\mathrm{CH}_{3} \mathrm{CN}$, are the most useful for this purpose as the relative line strengths are not affected by calibration and beam size differences.

Line strengths $T_{\mathrm{R}}^{*}$ in local thermal equilibrium are a function of kinetic temperature $T_{\text {kin }}$, molecular column density $N_{\text {mol }}$ and source size $\theta_{\mathrm{S}}$ given by the following equations:

$T_{\mathrm{R}}^{*}($ peak $)=\frac{\theta_{\mathrm{S}}^{2}}{\theta_{\mathrm{S}}^{2}+\theta_{\mathrm{B}}^{2}} T_{\mathrm{kin}}\left(1-\mathrm{e}^{-\tau}\right)$,

where $\theta_{\mathrm{B}}$ is the telescope beam FWHM and the optical depth at line peak $\tau$ is a function of $T_{\text {kin }}$ and sourceaveraged column density $N_{\text {mol }}$,

$\tau=\frac{2 \pi^{2}}{3 \epsilon_{0} k}\left(S \mu^{2} g_{\mathrm{I}} g_{\mathrm{K}}\right) 2 \sqrt{\frac{\log 2}{\pi}} \frac{\nu}{\Delta v}\left(\frac{N_{\mathrm{mol}} \mathrm{e}^{-E_{\mathrm{u}} / k T_{\text {kin }}}}{T_{\mathrm{kin}} Q\left(T_{\mathrm{kin}}\right)}\right)$.

Here, $\Delta v$ is the velocity FWHM for the molecule and transition under consideration, $k$ is the Boltzmann constant and $\epsilon_{0}$ the dielectric constant, $\nu$ and $E_{\mathrm{u}}$ are the rest frequency and upper level energy of the transition, $S$ is the line strength, $\mu$ the permanent dipole moment and $g_{\mathrm{I}}, g_{\mathrm{K}}$ are the reduced nuclear spin degeneracy and K-level degeneracy respectively. The constants are correct for S.I. units. The partition function $Q\left(T_{\text {kin }}\right)$ used here follows the high-temperature approximations for each molecule type given by Turner (1991). These approximations are valid for $k T_{\text {kin }} / h \ll B$ (or the largest rotation constant), which for the molecules seen in our survey is the case for temperatures $\geq 20 \mathrm{~K}$.

For molecules with multiple observed transitions, our first approach is to make rotation diagrams. This is a graphical method of estimating temperature and beamaveraged column density (e.g. Turner 1991) based on the relationship between column density, temperature and measured line intensity given in Eq. (1). The rotation diagram method further assumes that the line emission is optically thin, i.e. $\tau$ is small and $\left(1-\mathrm{e}^{-\tau}\right) \simeq \tau$. In this case, Eqs. (1) and (2) reduce to the single equation

$N_{\text {mol }}=\frac{3 k \epsilon_{0}}{2 \pi^{2}} \frac{\int T_{\mathrm{R}}^{*} \mathrm{~d} v}{\nu S \mu^{2} g_{\mathrm{I}} g_{\mathrm{K}}} Q\left(T_{\text {kin }}\right) \mathrm{e}^{E_{\mathrm{u}} / k T_{\text {kin }}}$.

Writing $L=3 k_{\mathrm{B}} \epsilon_{0} \int T_{\mathrm{R}}^{*} \mathrm{~d} v / 2 \pi^{2} \nu S \mu^{2} g_{\mathrm{I}} g_{\mathrm{K}}$ and $T_{\text {rot }}=T_{\text {kin }}$, Eq. (3) can be rearranged to give

$\log _{10} L=\log _{10}\left(\frac{N_{\text {mol }}}{Q\left(T_{\text {rot }}\right)}\right)-\frac{E_{\mathrm{u}}}{k} \frac{\log _{10} \mathrm{e}}{T_{\text {rot }}}$,

which on a plot of $\log _{10} L$ vs. $E_{\mathrm{u}} / k$ is a straight line with gradient of $-\left(\log _{10} \mathrm{e}\right) / T_{\text {rot }}$ and y-intercept of $\log _{10}\left(N_{\text {mol }} / Q\left(T_{\text {rot }}\right)\right) . N_{\text {mol }}$ and $T_{\text {rot }}$ are determined by a least-squares straight line fit to the data. Rotation diagrams are appropriate where emission is optically thin and from a source in thermal equilibrium which is characterised by a single temperature and density.

Secondly, we model line strengths using a full local thermal equilibrium (LTE) model which predicts line strengths as a function of kinetic temperature $T_{\text {kin }}$, sourceaveraged molecular column density $N_{\text {mol }}$, and source size $\theta_{\mathrm{S}}$ according to Eqs. (1) and (2) without assuming the optical depth $\tau$ is small.

These equations are then valid for transitions with significant optical depths as well as for optically thin lines, but still require that the lines are thermalised. Critical densities for many of our observed transitions of $\mathrm{CH}_{3} \mathrm{CN}$ and $\mathrm{CH}_{3} \mathrm{OH}$ are over $10^{6} \mathrm{~cm}^{-3}$, and these cores are one of the few places in which thermalization is a realistic possibility. For molecules such as $\mathrm{CH}_{3} \mathrm{CN}$, observations of lines from isotopomers imply that the optical depth is significant in the main line, and the rotation diagram method is not sufficient. Our LTE modelling approach is similar to the modified rotation diagram method used by Olmi et al. (1996b) for the hot core sources G10.47 and G31.41. We find that the rotation diagram method is highly unreliable when applied to small numbers of transitions, to transitions with significant optical depth, or to 
observations which sample more than one set of physical conditions along the line of sight. Throughout, we have obtained more consistent and convincing results using simple LTE models for the excitation. We note that the LTE model is fitting $T_{\mathrm{R}}^{*}$ directly rather than a logarithmic function of $T_{\mathrm{R}}^{*}$.

A third method of analysis would be to use a more advanced statistical equilibrium radiation transfer treatment as an alternative to our rotation diagram/LTE model approach. This type of approach was ruled out partly because of the extra time requirements of additional complexity, and partly because for the simple molecules we have observed too few lines, and for the complex species such as methanol and methyl cyanide the required data on collision cross-sections are unavailable for the high energy states. Comments on the validity of the LTE assumption are given for each molecule.

We estimated line parameters by fitting Gaussians or by taking linewidth and peak brightness directly from the spectra and using $\int T_{\mathrm{R}}^{*} \mathrm{~d} \nu \simeq 1.06 * T_{\mathrm{A}}^{*} \Delta \nu / \eta_{\mathrm{fss}}$. Where multiple transitions from the same molecule were found in a single spectrum, we fit the lines simultaneously with Gaussians of the same width, fixed at the known line frequencies.

For molecules with only one or two detected transitions we have employed a modification of the rotation diagram Eq. (3) to evaluate a lower limit to the beam-averaged column density, $N_{\text {min }}$. An excitation temperature for this equation is determined by evaluating the turning point of the temperature dependent part $\left(\mathrm{d} / \mathrm{d} T\left(Q\left(T_{\text {kin }}\right) \mathrm{e}^{E_{\mathrm{u}} / k T_{\text {kin }}}\right)=0\right)$. It may be shown that for linear molecules $T_{\text {kin }}=E_{\mathrm{u}} / k$ and for symmetric and asymmetric top molecules $T_{\text {kin }}=2 E_{\mathrm{u}} / 3 k$. Evaluating the second derivative shows that the turning point is a minimum and hence $N_{\min }$ can be found, as shown in Eqs. (5).

$N_{\min }=\frac{3 k \epsilon_{0}}{2 \pi^{2}} \frac{\int T_{\mathrm{R}}^{*} \mathrm{~d} v}{\nu S \mu^{2} g_{\mathrm{I}} g_{\mathrm{K}}} Q\left(E_{\mathrm{u}} / k\right) \mathrm{e}$,

for linear molecules,

$N_{\text {min }}=\frac{3 k \epsilon_{0}}{2 \pi^{2}} \frac{\int T_{\mathrm{R}}^{*} \mathrm{~d} v}{\nu S \mu^{2} g_{\mathrm{I}} g_{\mathrm{K}}} Q\left(2 E_{\mathrm{u}} / 3 k\right) \mathrm{e}^{2 / 3}$,

for symmetric and asymmetric tops.

Where we have not detected a molecule of interest, we have evaluated an upper limit to the beam-averaged column density $\left(N_{\max }\right)$. Our approach is to first determine a $3 \sigma$ detection limit for the line temperature $T_{\mathrm{R}}^{*}$ in order to find an upper limit to the detectable integrated intensity. An assumption of the gas temperature must also be made to evaluate $N_{\max }$ from Eq. (3).

Source geometry is a factor in the excitation models. In Sect. 4.1 we show that some of these sources have a dense, hot, chemically rich compact core and a halo of cooler gas. The hot core components are much smaller than the JCMT beam. Where this is the case, we take the hot gas to be concentrated centrally in one condensation (see discussion). We assume circular symmetry when es- timating source sizes and masses from the observed beam filling factors.

\subsection{Results by molecule}

We discuss the results molecule by molecule below, starting with the larger molecules with many lines in our frequency bands, and working down to molecules for which single lines only were observed.

\subsection{1. $\mathrm{CH}_{3} \mathrm{CN}$}

We observed the $J=19-18(349 \mathrm{GHz})$ and $13-$ $12(239 \mathrm{GHz})$ transitions of $\mathrm{CH}_{3} \mathrm{CN}$ (methyl cyanide). $\mathrm{CH}_{3} \mathrm{CN}$ is a symmetric top molecule with rotational levels split by the $K$ quantum number, with the different $K$ transitions for each $J+1 \rightarrow J$ closely grouped in frequency. We observed $K$ transitions from $K=0$ to 8 for $J=13-12$ and $K=0$ to 6 for $J=19-18$. Excitation energies of the observed lines range from 60 to $430 \mathrm{~K}$.

We detected $\mathrm{CH}_{3} \mathrm{CN}$ with a signal-to-noise ratio of more than two in eight sources: G5.89, G9.62, G10.47, G12.21, G29.96, G31.41, G34.26 and G75.78. We failed to detect any lines above $2 \sigma(\sim 0.1 \mathrm{~K})$ in six sources: G10.30, G13.87, G43.89, G45.12, G45.45 and G45.47. In G34.26, G10.47 and G31.41, we also detected $\mathrm{CH}_{3}^{13} \mathrm{CN}$ lines. These are weak and blended with the higher $K$ components of $\mathrm{CH}_{3} \mathrm{CN}$, but line strengths of up to $0.3 \mathrm{~K}$ are visible, and the existence of lines at all the expected $\mathrm{CH}_{3}^{13} \mathrm{CN}$ frequencies confirms the identification. At the $20^{\prime \prime}$ offset positions, we detected the $J=13-12 \mathrm{~K}=0-3$ lines of $\mathrm{CH}_{3} \mathrm{CN}$ towards G9.62. No other source observed at the offset position (G10.47, G29.96, G31.41 and G34.26) exhibited any methyl cyanide.

We first analysed the excitation using rotation diagrams, assuming the gas to be optically thin. Figure 2 shows the $\mathrm{CH}_{3} \mathrm{CN}$ data plotted in the rotation diagram format. Straight lines fit the data poorly, with attempts at a fit producing unexpectedly high rotation temperatures and positive rather than negative gradients in some cases. By changing the number of datapoints in the fit (removing lines at random) the rotation temperature varied widely, and no confidence could be placed in the results. We conclude that the rotation diagram method is unsuitable for the analysis of $\mathrm{CH}_{3} \mathrm{CN}$ in these objects. The assumptions on which it is based must break down indicating high optical depth, anomalous excitation, or source structure.

The LTE model including optical depth produces a better fit to the data, as shown in Fig. 3. In order to estimate line parameters for the LTE excitation model, we used a Gaussian fit, fitting to all $\mathrm{CH}_{3} \mathrm{CN}$ lines in each band simultaneously. We included in our fit the $K=0$ and 1 lines, which are blended with each other, as the Gaussian 

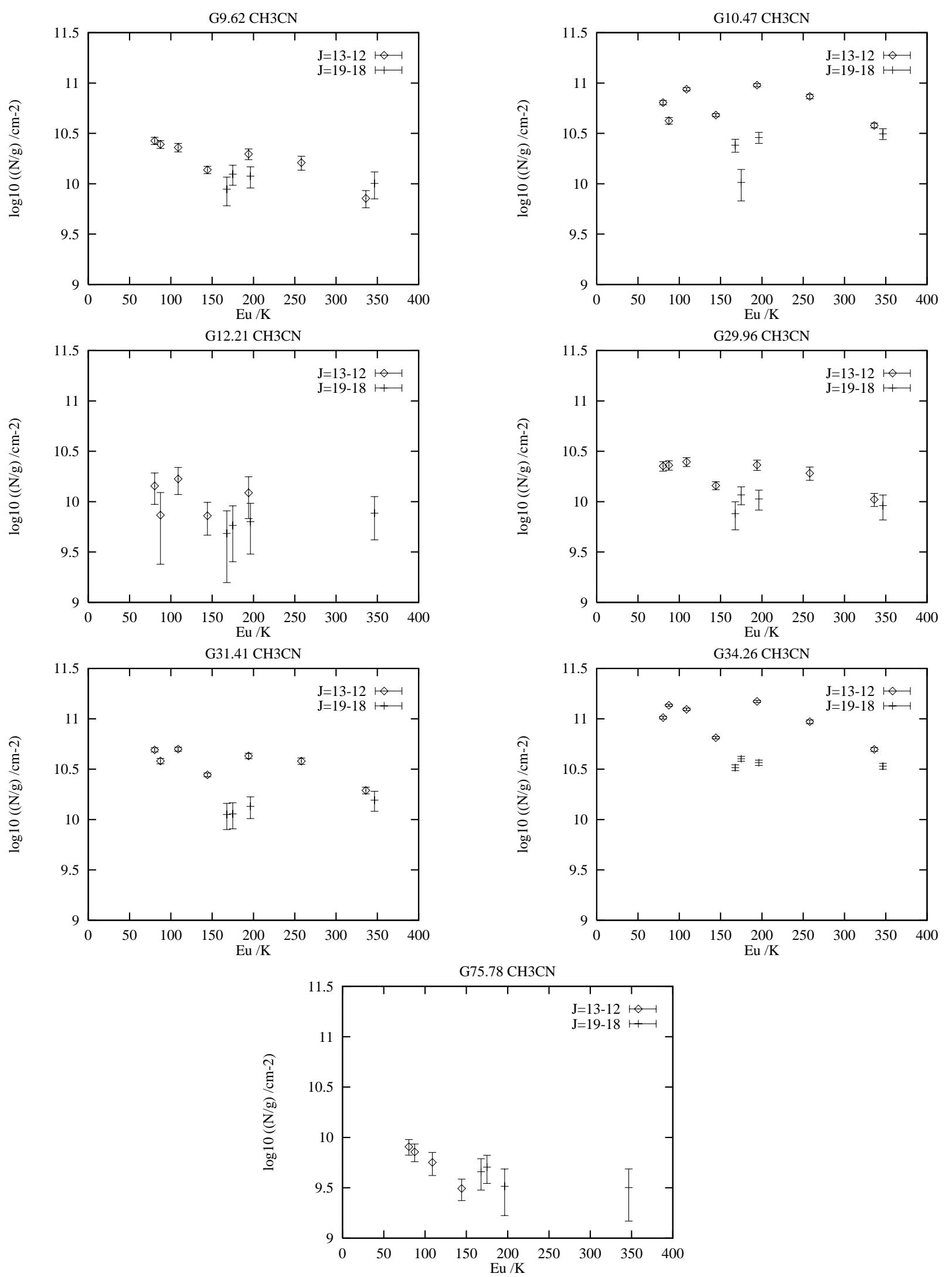

Fig. 2. $\mathrm{CH}_{3} \mathrm{CN}$ data in rotation diagram format. We were unable to fit convincing straight lines to these data 

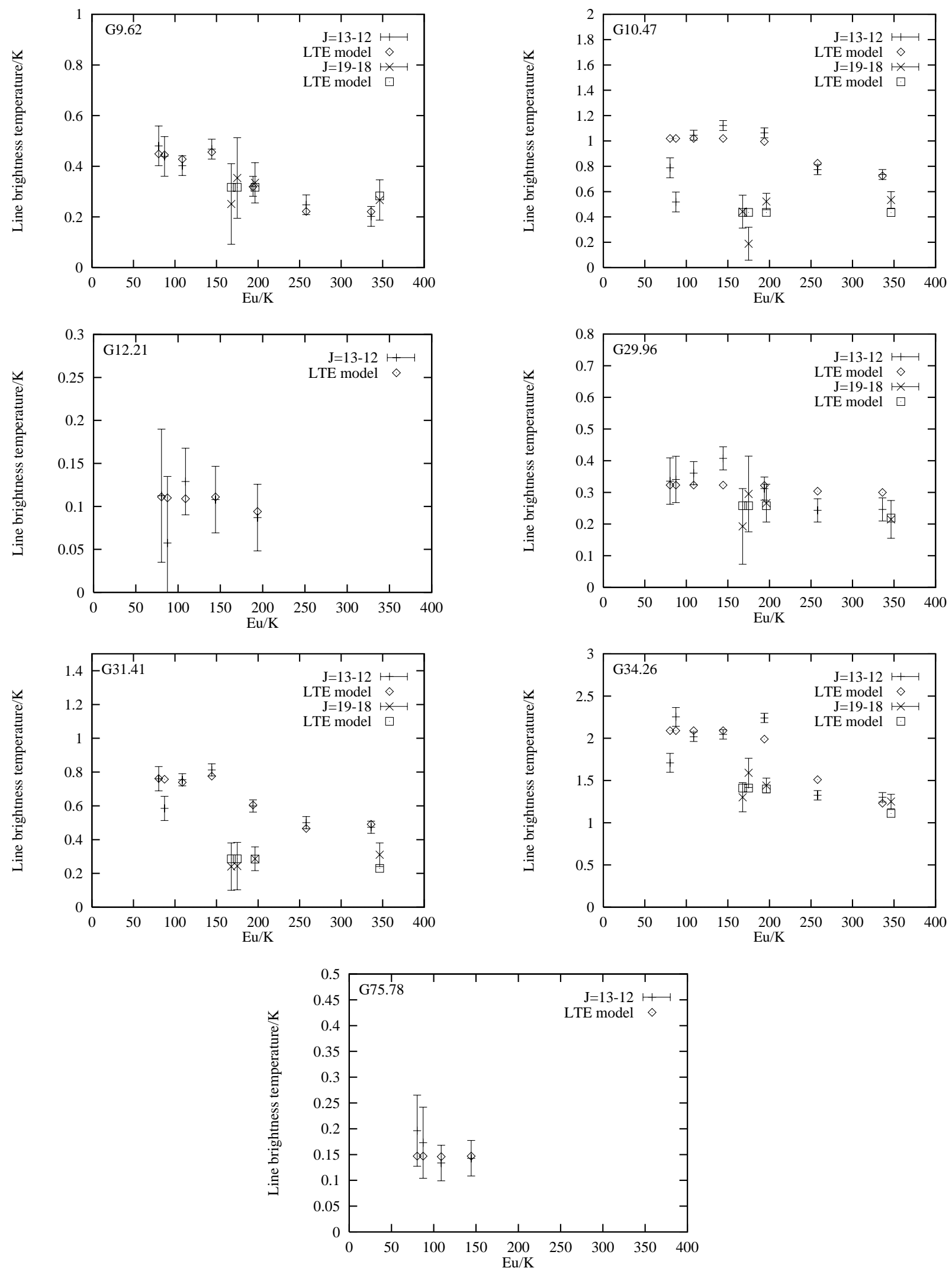

Fig. 3. $\mathrm{CH}_{3} \mathrm{CN}$ data overlaid with the predictions of the LTE model. The data are plotted as bars $(J=13-12)$ and crosses $(J=19-18)$ with errorbars, and the model predictions as diamonds and squares 
fit produces separate estimates for their strengths. We believe the measured strengths for these lines to be less accurate than for $K \geq 2$, so in the modelling below we artificially increased their uncertainties by a factor of two. We did not use the $J=19-18 K=3$ and 4 lines, as they are confused with $\mathrm{CCH}$ lines, and nor did we fit the $J=13-12 K \geq 7$ lines, which are confused with $\mathrm{HCOOCH}_{3}$ and $\mathrm{CH}_{3}^{13} \mathrm{CN}$. We include $J=13-12 \mathrm{~K}=5$ and 6 . These are also affected by blending with $\mathrm{CH}_{3}^{13} \mathrm{CN}$ but to a lesser extent than the higher $K$ lines. We also include $J=13-12 \mathrm{~K}=4$ although there is some contribution to this line from $\mathrm{SO}_{2}$ in the lower sideband. We did not analyse the G5.89 $J=19-18$ lines. These observations of G5.89 were made on a separate occasion with slightly different frequency bands, and the $\mathrm{CH}_{3} \mathrm{CN}$ lines, though clearly present at levels of up to $1 \mathrm{~K}$, fall across two spectra in the noisy extremities of the bands.

The $\mathrm{CH}_{3} \mathrm{CN}$ lines all have excitation energies of $>60 \mathrm{~K}$. Emission in these lines must be dominated by hot gas, such as we would expect to find in a compact core, and therefore a model with a single temperature and density is appropriate. We use a least- $\chi^{2}$ fit to choose the three parameters $\left(T, N_{\mathrm{CH}_{3} \mathrm{CN}}\right.$ and $\left.\theta_{\mathrm{S}}\right)$ that produce the closest match to the observed line intensities. To estimate the uncertainties on the estimates of $T_{\text {kin }}, N_{\mathrm{CH}_{3} \mathrm{CN}}$ and $\theta_{\mathrm{S}}$, we use a standard Monte-Carlo technique, adding random noise to the best-fit line intensities to create multiple synthetic datasets, which then produce a distribution of results for each parameter from which the uncertainties can be estimated.

Temperature and source size estimates are shown in Table 3. Uncertainties in the estimates from the $\mathrm{CH}_{3} \mathrm{CN}$ $J=19-18$ data are larger than for the $J=13-12$, reflecting the better signal-to-noise ratio of the $J=13-12$ observations. Not all parameters could be determined in all sources.

Column density is poorly determined by the excitation model, as is expected for significant optical depths. The line intensities are relatively insensitive to column density as it appears in the negative exponential for $\tau$ only (Eq. (1)), which for high $\tau$ varies only slowly. In Table 3 we give instead the beam-averaged column density lower limits (from the $E_{\mathrm{u}}=109 \mathrm{~K} J=13-12$ $K=2$ line). These considerably underestimate the true $\mathrm{CH}_{3} \mathrm{CN}$ column densities in the core, as the core is much smaller than the beam (Table 3 ). In the strongest sources the excitation model fits require $\mathrm{CH}_{3} \mathrm{CN}$ column densities of at least $10^{16} \mathrm{~cm}^{-2}$. In the three sources in which $\mathrm{CH}_{3}^{13} \mathrm{CN}$ is detected (G10.47, G31.41 and G34.26) the $\mathrm{CH}_{3}^{13} \mathrm{CN} / \mathrm{CH}_{3}^{12} \mathrm{CN}$ line ratios imply optical depths of order 10 in the $J=13-12 \mathrm{~K}=3-5$ lines, or $\mathrm{CH}_{3} \mathrm{CN}$ column densities of at least $10^{17} \mathrm{~cm}^{-2}$, taking $\left[{ }^{12} \mathrm{C}\right] /\left[{ }^{13} \mathrm{C}\right]$ $\simeq 50$. These column densities are consistent with the observed line intensities in $\mathrm{CH}_{3} \mathrm{CN}$ and the results of the LTE fit.
We find gas temperatures between 70 and $200 \mathrm{~K}$. These high temperatures are required to excite lines with excitation energies of several hundred kelvin.

The size of the $\mathrm{CH}_{3} \mathrm{CN}$ emitting region is small $(D<$ $0.1 \mathrm{pc}$ ) in all sources. In the sources with the best signalto-noise, G34.26 and 10.47, the source sizes suggest that the lower-excitation $J=13-12$ lines come from a larger region than the $J=19-18$. The results for the other sources (for which the uncertainties on source size are greater) are also consistent with this suggestion. This is indirect evidence for a temperature or density gradient, as the $J=19-18$ lines have higher critical densities and higher excitation energies.

There is no direct evidence from $\mathrm{CH}_{3} \mathrm{CN}$ for temperature or column density differences between sources, once uncertainties are taken into account. This is partly because the uncertainties of the temperature and sourceaveraged column density estimates are large. Also, the optical depth in the $\mathrm{CH}_{3} \mathrm{CN}$ transitions is significant, so most of the emission we receive comes from the front of the $\mathrm{CH}_{3} \mathrm{CN}$ emitting region. In all sources, this will be the nearest region with sufficient temperature and density to excite the lines, ie. approximately the critical density. The size of the hot, compact core from which the $\mathrm{CH}_{3} \mathrm{CN}$ lines are emitted appears to vary between sources, with the strongest emission from the largest sources.

Beam-averaged column density upper limits towards the sources with no detected methyl cyanide (G10.30, G13.87, G43.89, G45.21, G45.45 and G45.47) were evaluated and found to be between $8-910^{12} \mathrm{~cm}^{-2}$. Upper limits were calculated assuming a gas temperature of $50 \mathrm{~K}$ and an average linewidth of $6 \mathrm{MHz}$.

Upper limits for the $20^{\prime \prime}$ offset positions were calculated, giving $\mathrm{CH}_{3} \mathrm{CN}$ column densities (beam-averaged) of less than $1.110^{13} \mathrm{~cm}^{-2}$. $\mathrm{CH}_{3} \mathrm{CN}$ was detected at the offset position in G9.62 with a beam-averaged column density lower limit of $1.010^{13} \mathrm{~cm}^{-2}$.The temperature assumed for the offset position was $50 \mathrm{~K}$, based on the temperature derived from the $\mathrm{CH}_{3} \mathrm{CCH}$ rotation diagram at this position.

The estimates for G10.47 and G31.41 are consistent with the interferometer results and observations of vibrationally excited $\mathrm{CH}_{3} \mathrm{CN}$ (Olmi et al. 1996a,b). Temperatures are generally higher than those calculated from lower- $J$ transitions of $\mathrm{CH}_{3} \mathrm{CN}$ and from ammonia (Olmi et al. 1993).

Virial masses calculated from $\mathrm{CH}_{3} \mathrm{CN}$ are given in Table 3. These are typically a few hundred solar masses. For the strongest sources, by assuming virial equilibrium we estimate $N_{\mathrm{H}_{2}} \simeq 10^{25} \mathrm{~cm}^{-2}$ and $n_{\mathrm{H}_{2}} \simeq 10^{7}-10^{8} \mathrm{~cm}^{-3}$, which is sufficient for the gas to be thermalised. Working again from the assumption of virial equilibrium, and taking $N_{\mathrm{CH}_{3} \mathrm{CN}}=10^{17} \mathrm{~cm}^{-2}$, we estimate a $\mathrm{CH}_{3} \mathrm{CN}$ abundance of $X_{\mathrm{CH}_{3} \mathrm{CN}} \simeq 10^{-8}$. 
Table 3. $\mathrm{CH}_{3} \mathrm{CN}$ results: temperature, angular diameter and distance-corrected source size for LTE model fits to the $J=19-18$ and $J=13-12$ transitions; line width, virial mass and implied $\mathrm{H}_{2}$ column density (averaged over source, from virial mass) from the $J=13-12$ transitions, and beam-averaged column density lower limits from the $J=13-12 \mathrm{~K}=2$ line

\begin{tabular}{|c|c|c|c|c|c|c|c|c|c|c|}
\hline \multirow[b]{2}{*}{ Source } & \multicolumn{2}{|c|}{$\begin{array}{c}T \\
{[\mathrm{~K}]}\end{array}$} & \multicolumn{2}{|c|}{$\begin{array}{l}\theta_{\mathrm{S}} \\
{\left[{ }^{\prime \prime}\right]}\end{array}$} & \multicolumn{2}{|c|}{$\begin{array}{c}D \\
{[\mathrm{pc}]}\end{array}$} & \multirow[t]{2}{*}{$\begin{array}{c}\Delta v \\
{\left[\mathrm{~km} \mathrm{~s}^{-1}\right]}\end{array}$} & \multirow[t]{2}{*}{$\begin{array}{l}M_{\text {vir }} \\
{\left[M_{\odot}\right]}\end{array}$} & \multirow[t]{2}{*}{$\begin{array}{c}N_{\mathrm{H}_{2}} \\
{\left[10^{24} \mathrm{~cm}^{-2}\right]}\end{array}$} & \multirow[t]{2}{*}{$\begin{array}{c}N_{\mathrm{CH}_{3} \mathrm{CN}} \\
{\left[10^{14} \mathrm{~cm}^{-2}\right]}\end{array}$} \\
\hline & $13-12$ & $19-18$ & $13-12$ & $19-18$ & $13-12$ & $19-18$ & & & & \\
\hline G9.62 & $119_{-43}^{+62}$ & $65_{-42}^{+189}$ & $1.19_{-0.07}^{+0.90}$ & $0.98_{-0.69}^{+0.26}$ & 0.033 & 0.027 & 7.5 & 190 & 12 & $>0.2$ \\
\hline G10.47 & $87_{-16}^{+14}$ & $134_{-113}^{+161}$ & $2.29_{-0.41}^{+0.41}$ & $0.80_{-0.40}^{+0.30}$ & 0.064 & 0.022 & 11 & 810 & 14 & $>1.3$ \\
\hline G12.21 & - & - & - & - & - & - & 17 & - & - & $>0.2$ \\
\hline G29.96 & $114_{-80}^{+140}$ & $141_{-121}^{+360}$ & $1.12_{-0.52}^{+0.76}$ & $0.60_{-0.24}^{+1.38}$ & 0.040 & 0.021 & 9.0 & 330 & 15 & $>0.3$ \\
\hline G31.41 & $149_{-36}^{+27}$ & $142_{-119}^{+199}$ & $1.53_{-0.25}^{+0.34}$ & $0.63_{-0.31}^{+1.24}$ & 0.058 & 0.024 & 8.7 & 450 & 10 & $>0.5$ \\
\hline G34.26 & $79_{-8}^{+8}$ & $141_{-42}^{+34}$ & $3.46_{-0.55}^{+0.55}$ & $1.41_{-0.20}^{+0.34}$ & 0.066 & 0.027 & 8.1 & 460 & 7 & $>1.1$ \\
\hline G75.78 & - & - & - & - & - & - & 5.5 & - & - & $>0.1$ \\
\hline
\end{tabular}

\subsection{2. $\mathrm{CH}_{3} \mathrm{CCH}$}

The $\mathrm{CH}_{3} \mathrm{CCH}$ (propyne) $J=14-13$ lines lie in the same passband as the $\mathrm{CH}_{3} \mathrm{CN} J=13-12$ lines at $239 \mathrm{GHz}$. $\mathrm{CH}_{3} \mathrm{CCH}$ is again a symmetric top molecule with several transitions closely spaced in frequency. The $K=0$ to 3 components were observed in most sources. The $K=0$ and $K=1$ lines are blended with each other and the $K=$ 2 line is often blended with a line of $\mathrm{CH}_{3} \mathrm{CHO}$, precluding the use of rotation diagrams.

$\mathrm{CH}_{3} \mathrm{CCH}$ was detected towards 10 of our 14 sources; G9.62, G10.30, G10.47, G12.21, G13.87, G29.96, G31.41, G34.26, G45.47 and G75.78. The low dipole moment (0.78 Debye) leads to fairly easy thermalisation of $\mathrm{CH}_{3} \mathrm{CCH}$ and the fairly weak line temperatures $\left(T_{\mathrm{R}}^{*}<1 \mathrm{~K}\right)$ suggests a low optical depth. The excitation energies of the $K=0$ to 4 lines range from 90 to $200 \mathrm{~K}$ and they require hot gas for excitation. We note that two of these sources were not detected in $\mathrm{CH}_{3} \mathrm{CN}$ transitions with lower $E_{\mathrm{U}}$.

Lower limits for the beam-averaged column density have been evaluated for the sources in which $\mathrm{CH}_{3} \mathrm{CCH}$ was detected and upper limits for the beam-averaged column density have been evaluated in 3 sources where $\mathrm{CH}_{3} \mathrm{CCH}$ has not been detected (G43.89, G45.12 and G45.45), and these are given in Table 4. Upper limits were calculated assuming a gas temperature of $50 \mathrm{~K}$ and a linewidth of $6 \mathrm{MHz}$. Note that the remaining source G5.89 was not observed at the $239 \mathrm{GHz}$ band.

The beam-averaged column density of $\mathrm{CH}_{3} \mathrm{CCH}$ is fairly similar towards most of the sources, apart from the two most line-rich sources and two of the line-poor sources. The column density lower limits of $\mathrm{CH}_{3} \mathrm{CCH}$ towards G34.26 and G10.47 are a factor of 5-6 times greater than the other sources. The sources exhibiting low column densities of $\mathrm{CH}_{3} \mathrm{CCH}$ are $\mathrm{G} 45.47$ and G13.87, the two linepoor sources for which we have $\mathrm{CH}_{3} \mathrm{CCH}$ detections rather than upper limits.

We have also detected $\mathrm{CH}_{3} \mathrm{CCH}$ at $20^{\prime \prime}$ offset positions in two sources (G34.26 and G9.62). The offset positions should characterise the conditions in the halo. The $K=$ 0 to 3 components were detected at both these offsets,
Table 4. $\mathrm{CH}_{3} \mathrm{CCH}$ beam-averaged column density upper and lower limits

\begin{tabular}{|c|c|}
\hline Source & $N_{\mathrm{mol}}\left[10^{14} \mathrm{~cm}^{-2}\right]$ \\
\hline G9.62 & $>4.6$ \\
\hline G10.30 & $>3.0$ \\
\hline G10.47 & $>18.6$ \\
\hline G12.21 & $>3.3$ \\
\hline G13.87 & $>1.4$ \\
\hline G29.96 & $>5.4$ \\
\hline G31.41 & $>4.1$ \\
\hline G34.26 & $>15.8$ \\
\hline G43.89 & $<3.3$ \\
\hline G45.12 & $<3.1$ \\
\hline G45.45 & $<3.1$ \\
\hline G45.47 & $>2.2$ \\
\hline G75.78 & $>2.9$ \\
\hline
\end{tabular}

with narrow enough linewidths to separate the $K=0$ and 1 components blended at the central position. From a rotation diagram analysis of the offset positions, the halo component towards G34.26 has a rotation temperature of $38 \pm 3 \mathrm{~K}$ and a column density of $4.6 \pm 0.710^{14} \mathrm{~cm}^{-2}$. The halo component towards G9.62 has a slightly higher rotation temperature of $46 \pm 10 \mathrm{~K}$ and a slightly lower beam-averaged column density of $2.6 \pm 1.010^{14} \mathrm{~cm}^{-2}$.

Comparing $\mathrm{CH}_{3} \mathrm{CCH}$ column densities of a few $\times 10^{14} \mathrm{~cm}^{-2}$ with the $\mathrm{H}_{2}$ hot core column densities from $\mathrm{CH}_{3} \mathrm{CN}$ (above) gives fractional abundances of a few times $10^{-11}$. This abundance estimate is a lower limit on the true abundance as the column densities in G34.26 and G9.62 at the offset positions are similar to the on-source positions, which suggests that the $\mathrm{CH}_{3} \mathrm{CCH}$ is excited over a larger region than $\mathrm{CH}_{3} \mathrm{CN}$. $\mathrm{CH}_{3} \mathrm{CCH}$ emission traces hot gas and is likely to come from a more compact, denser region than the $\mathrm{CO}$.

\subsection{3. $\mathrm{CH}_{3} \mathrm{OH}$}

We observed lines of $\mathrm{CH}_{3} \mathrm{OH}$ (methanol) with frequencies in the 216, 241, 337, 346 and $349 \mathrm{GHz}$ bands. 
Table 5. $\mathrm{CH}_{3} \mathrm{OH}$ hot core component results: temperature, source-averaged column density, source diameter, distance, FWHM, virial mass, $\mathrm{H}_{2}$ source-averaged column density (from virial mass) and fractional abundance

\begin{tabular}{lcccccccc}
\hline Source & $\begin{array}{c}T \\
{[\mathrm{~K}]}\end{array}$ & $\begin{array}{c}N_{\mathrm{CH}_{3} \mathrm{OH}} \\
{\left[10^{17} \mathrm{~cm}^{-2}\right]}\end{array}$ & $\begin{array}{c}\theta_{\mathrm{S}} \\
{\left[{ }^{\prime \prime}\right]}\end{array}$ & $\begin{array}{c}D \\
{[\mathrm{pc}]}\end{array}$ & $\begin{array}{c}\Delta v \\
{\left[\mathrm{~km} \mathrm{~s}^{-1}\right]}\end{array}$ & $\begin{array}{c}M_{\text {vir }} \\
{\left[M_{\odot}\right]}\end{array}$ & $\begin{array}{c}N_{\mathrm{H}_{2}} \\
{\left[10^{24} \mathrm{~cm}^{-2}\right]}\end{array}$ & $\begin{array}{c}X_{\mathrm{CH}_{3} \mathrm{OH}} \\
{\left[10^{-8}\right]}\end{array}$ \\
\hline G9.62 & $67_{-23}^{+57}$ & $2.4_{-1.0}^{+3.1}$ & $1.6_{-0.4}^{+0.4}$ & 0.044 & 7.4 & 250 & 9 & 3 \\
G10.47 & $52_{-6}^{+8}$ & $3.1_{-0.7}^{+1.1}$ & $3.1_{-0.3}^{+0.1}$ & 0.086 & 11 & 1100 & 10 & 3 \\
G12.21 & $56_{-24}^{+800}$ & $3.9_{-2.2}^{+300}$ & $1.3_{-1.0}^{+0.5}$ & 0.084 & 12 & 1300 & 12 & 3 \\
G29.96 & $48_{-11}^{+17}$ & $4.2_{-1.4}^{+4.0}$ & $2.0_{-0.3}^{+0.3}$ & 0.071 & 5.8 & 250 & 3 & 14 \\
G31.41 & $54_{-10}^{+13}$ & $1.7_{-0.7}^{+1.4}$ & $2.7_{-0.3}^{+0.3}$ & 0.102 & 8.7 & 820 & 5 & 3 \\
G34.26 & $54_{-2}^{+3}$ & $3.1_{-0.2}^{+0.3}$ & $4.5_{-0.1}^{+0.2}$ & 0.086 & 8.1 & 600 & 5 & 6 \\
G75.78 & $56_{-25}^{+79}$ & $2.1_{-1.0}^{+11}$ & $1.4_{-0.5}^{+0.5}$ & 0.028 & 5.1 & 80 & 7 & 3 \\
\hline
\end{tabular}

The excitation energies of these lines range from $30 \mathrm{~K}$ to $800 \mathrm{~K}$. The higher excitation energy lines, in the $337 \mathrm{GHz}$ frequency range, are $v_{\mathrm{t}}=2$ vibrationally excited states of $\mathrm{CH}_{3} \mathrm{OH}$. Of ground state lines with excitation energy above $150 \mathrm{~K}$, we observed the 14(1) $-14(0) \pm E_{\mathrm{u}}=$ $260 \mathrm{~K}$ transition at $349.104 \mathrm{GHz}$ and the $12(1)-12(0)$ $\pm E_{\mathrm{u}}=190 \mathrm{~K}$ transition at $336.865 \mathrm{GHz}$. We observed many lines with excitation energies of less than $150 \mathrm{~K}$.

We detected $\mathrm{CH}_{3} \mathrm{OH}$ in all sources. The $12(1)-12(0)$ \pm and $14(1)-14(0) \pm$ transitions were observed in G5.89, G9.62, G12.21, G10.47, G29.96, G31.41, G34.26, and G75.78. Vibrationally excited lines and ${ }^{13} \mathrm{CH}_{3} \mathrm{OH}$ lines were only observed in G34.26, G10.47 and G31.41. $\mathrm{CH}_{3} \mathrm{OH}$ lines were detected at the $20^{\prime \prime}$ offset positions in all five sources where these were observed. The ${ }^{13} \mathrm{CH}_{3} \mathrm{OH}$ lines are confused with $\mathrm{CH}_{3} \mathrm{CCH}, \mathrm{HCOOCH}_{3}$ and other lines but are clearly present in these three sources with line strengths of a few times $0.1 \mathrm{~K}$.

To estimate the line parameters, we fit Gaussians simultaneously to groups of lines with similar frequency. In the $337 \mathrm{GHz}$ band, there are many vibrational lines of $\mathrm{CH}_{3} \mathrm{OH}$ and it is difficult to separate and identify individual lines, but we estimate line strengths of up to $1 \mathrm{~K}$ in these transitions.

As in the $\mathrm{CH}_{3} \mathrm{CN}$ case, rotation diagram analysis failed to produce satisfactory results. The fits to the data were generally poor. When A-type and E-type $\mathrm{CH}_{3} \mathrm{OH}$ were analysed separately, derived rotation temperatures differed by a factor of more than four in every source. The observed $\mathrm{CH}_{3} \mathrm{OH}$ lines span a wide range of excitation energies (from 30 to more than $500 \mathrm{~K}$ ) and trace both hot and cold gas, so that the emission cannot be fitted with a single straight line corresponding to a single temperature component, and this plus moderate optical depths is why the rotation diagram method fails.

Evidence from other molecules such as $\mathrm{CH}_{3} \mathrm{CN}$ and $\mathrm{CO}$ which show a hot, dense compact core and a cooler halo component leads us to try a two-component LTE model for the seven sources which show $E_{\mathrm{u}}>150 \mathrm{~K}$ lines. This produces a reasonable fit to the ground state $\mathrm{CH}_{3} \mathrm{OH}$ lines. In the two-component model, the line emission is assumed to originate from two regions, a hot core and a cool halo, each characterized by temperature, $\mathrm{CH}_{3} \mathrm{OH}$ column density, and source size.

$E_{\mathrm{u}}>100 \mathrm{~K}$ states are populated almost entirely by the hot core component and we use these to determine its physical parameters. Using the ratios between the $E_{\mathrm{u}}=115,197$ and $260 \mathrm{~K}$ lines $(J(K)=5(4)-6(3) \mathrm{A}$, $12(1)-12(0)$ and $14(1)-14(0))$ we estimate $N_{\mathrm{CH}_{3} \mathrm{OH}}$ and $T_{\text {kin }}$ using Eqs. (3) and (4). The line strength in the $E_{\mathrm{u}}=197 \mathrm{~K}$ line, which is the strongest, is then used to estimate the core size. Results for the hot core are given in Table 5 and for the cold halo in Table 6 . The cold halo component is assumed to have an angular size sufficient to fill the telescope beam and a temperature of $15 \mathrm{~K}$, and we estimate the beam-averaged column density from the $E_{\mathrm{u}}=35 \mathrm{~K}$ line.

For the six sources without $E_{\mathrm{u}}>100 \mathrm{~K}$ lines, beamaveraged column density lower limits were estimated from the observed low $E_{\mathrm{u}}$ lines, and these are also given in Table 6. Again because of reduced frequency coverage we were unable to make an analysis for G5.89, but the strengths of the $14(1)-14(0)$ and $12(1)-12(0)$ transitions indicate that it too has a hot $\mathrm{CH}_{3} \mathrm{OH}$ core. The beam-averaged column density lower limits in these sources probably do not underestimate the true column densities by much, as the emission is likely to be extended (as in the sources for which the source size was determined) and at a similar temperature to that for which the lower limit was determined, $32 \mathrm{~K}$.

We could not fit the $v_{\mathrm{t}}=2$ lines, which have excitation energies of $>500 \mathrm{~K}$, with any LTE model. These were observed in the three richest sources G34.26, G10.47 and G31.41. These lines can be excited by infrared emission from hot dust at a wavelength of $\sim 25 \mu \mathrm{m}$ (Lovas et al. 1982). Vibrational transitions of $\mathrm{CH}_{3} \mathrm{CN}$ were observed by Olmi et al. (1996b) in G31.41 and G10.47 and may be excited by similar infrared wavelengths, and high excitation transitions of CS in G34.26 may also be excited in this way (Goldsmith et al. 1983; Hauschildt et al. 1993). To excite these lines requires a dust temperature of several hundred kelvin, and this emission must come from a small region surrounding the exciting source.

To produce the observed ${ }^{13} \mathrm{CH}_{3} \mathrm{OH}$ line strengths of $\sim 0.2 \mathrm{~K}$ in G34.26, G31.41 and G10.47, an optically thick 

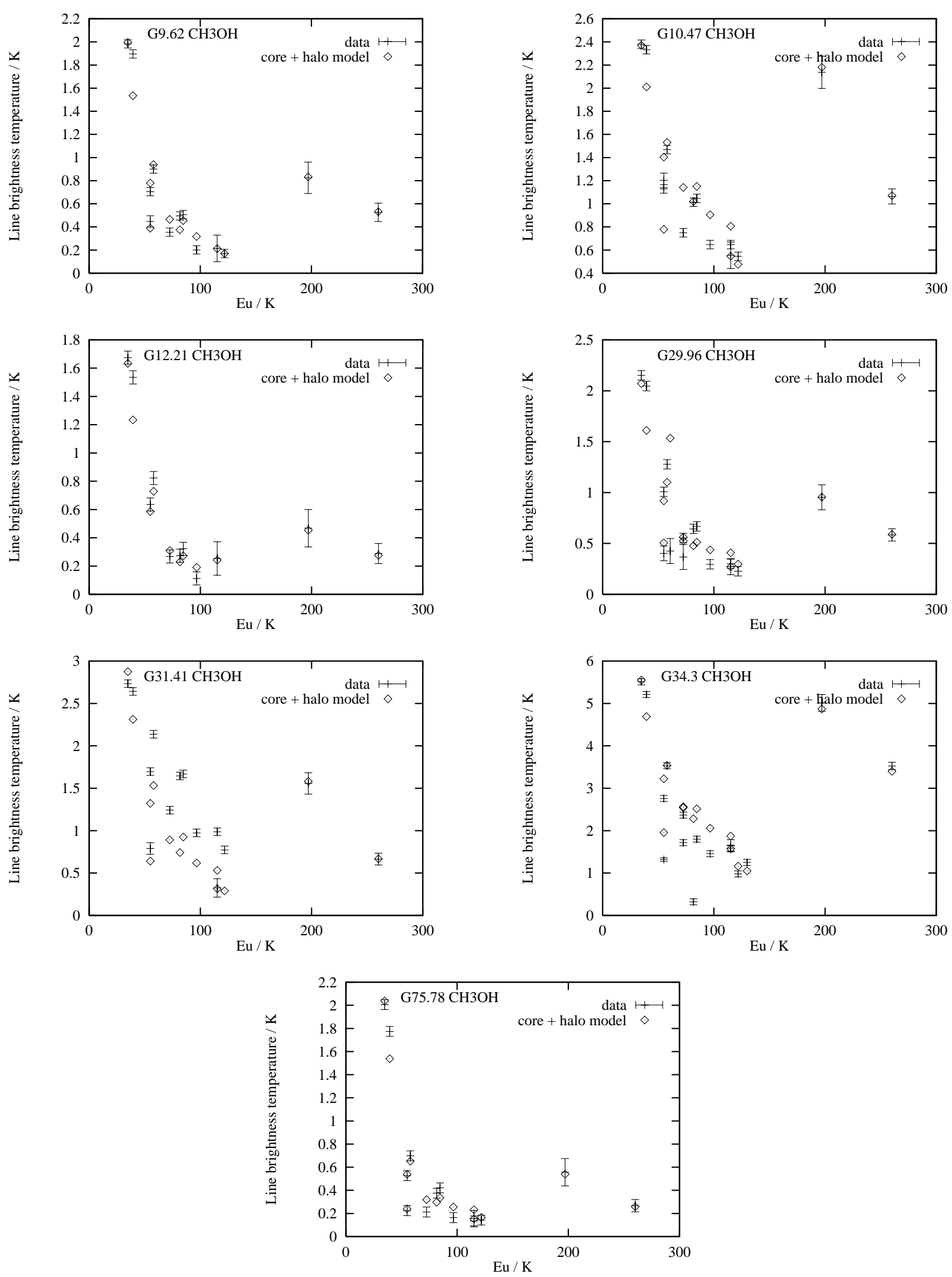

Fig. 4. $\mathrm{CH}_{3} \mathrm{OH}$ data (bars with errorbars) overlaid with the predictions of the LTE hot core plus cold halo model (diamonds) 
Table 6. $\mathrm{CH}_{3} \mathrm{OH}$ cold halo component column densities. Source-averaged column density estimates are from core plus halo model. Beam-averaged column density lower limits are given for the five sources for which we did not detect high excitation lines. Fractional abundances are calculated using $\mathrm{H}_{2}$ column densities estimated from $\mathrm{CO}$ for sources where we have estimates of both $N_{\mathrm{CH}_{3} \mathrm{OH}}$ and $N_{\mathrm{H}_{2}}$

\begin{tabular}{|c|c|c|}
\hline Source & $N_{\mathrm{CH}_{3} \mathrm{OH}}\left[10^{15} \mathrm{~cm}^{-2}\right]$ & $X_{\mathrm{CH}_{3} \mathrm{OH}}\left[10^{-10}\right.$ \\
\hline G9.62 & $\overline{0.7}$ & 6 \\
\hline G10.30 & 0.5 & - \\
\hline G10.47 & 0.8 & 9 \\
\hline G12.21 & 0.9 & 2 \\
\hline G13.87 & $>0.2$ & - \\
\hline G29.96 & 0.7 & 10 \\
\hline G31.41 & 1.0 & 14 \\
\hline G34.26 & 1.8 & 5 \\
\hline G43.89 & $>0.2$ & - \\
\hline G45.12 & $>0.1$ & - \\
\hline G45.45 & $>0.1$ & - \\
\hline G45.47 & $>0.4$ & - \\
\hline G75.78 & 0.4 & 4 \\
\hline
\end{tabular}

ultracompact core is required in addition to the compact component in the core-halo model. The $0.1 \mathrm{pc}$ compact core component in the LTE model with column densities of a few times $10^{17} \mathrm{~cm}^{-2}$ cannot account for the ${ }^{13} \mathrm{CH}_{3} \mathrm{OH}$ line brightness temperatures, which require optical depths greater than 10 in the corresponding ${ }^{12} \mathrm{CH}_{3} \mathrm{OH}$ lines, whereas the hot component in the core-halo model produces optical depths of less than 5 . We can estimate the properties of the optically thick ultracompact core as follows. Assuming an excitation temperature of $\sim 100 \mathrm{~K}$ producing line brightness temperatures of $0.2 \mathrm{~K}$, we estimate its size to be $\sim 1^{\prime \prime}$ or $0.02 \mathrm{pc}$ at $5 \mathrm{kpc}$ (in the optically thick case, $\left.T_{\mathrm{R}}^{*} \simeq \theta_{\mathrm{S}}^{2} /\left(\theta_{\mathrm{S}}^{2}+\theta_{\mathrm{B}}^{2}\right) T_{\mathrm{ex}}\right)$. This size is comparable to the source sizes seen in $\mathrm{CH}_{3} \mathrm{CN} J=19-18$. The source-averaged methanol column density must be at least $10^{18} \mathrm{~cm}^{-2}$ to give the observed ${ }^{13} \mathrm{CH}_{3} \mathrm{OH}$ line strengths. If methanol fractional abundances are $310^{-8}$ as in the compact core (see below) then hydrogen column densities in the ultracompact core are over $10^{26} \mathrm{~cm}^{-2}$ and gas densities are over $10^{9} \mathrm{~cm}^{-3}$.

The $\mathrm{CH}_{3} \mathrm{OH}$ transitions have critical densities up to more than $10^{6} \mathrm{~cm}^{-3}$. This condition is probably fulfilled in the compact core, but in the halo region subthermal excitation seems probable. However, if the populations were subthermal we would expect underpopulation in the higher- $K$ transitions, which have higher critical densities, and we see no evidence for this.

The hot core temperature, $\sim 50 \mathrm{~K}$, falls in between the values derived from $\mathrm{CH}_{3} \mathrm{CN}$ and $\mathrm{CO}$, suggesting that the $\mathrm{CH}_{3} \mathrm{OH}$ is tracing an intermediate volume of gas (Table 5). The temperature varies little from source to source, suggesting that the observed lines select for gas at this temperature, probably because of optical depth effects (the transitions have optical depths of a few). $\mathrm{CH}_{3} \mathrm{OH}$ hot core column densities and source sizes differ by a factor of up to 3 . In most cases the smaller sources have lower column densities (exceptions are G12.21 and G29.96). Absolute diameters for the compact core components are also shown in Table 5. G75.78 has the smallest hot $\mathrm{CH}_{3} \mathrm{OH}$ core, and G10.47, G34.26, G31.41 and G12.21 have the largest.

The virial mass, resultant $\mathrm{H}_{2}$ column density (sourceaveraged) and $\mathrm{CH}_{3} \mathrm{OH}$ fractional abundances for the $\mathrm{CH}_{3} \mathrm{OH}$ cores are given in Table 5 . We use the linewidth of the $E_{\mathrm{u}}=197 \mathrm{~K} J(K)=12(1)-12(0)$ line for the hot component. Virial masses range from $100-1300 M_{\odot}$ and $\mathrm{H}_{2}$ column densities from $3-1210^{24} \mathrm{~cm}^{-2}$. The fractional abundances given are the ratio of $\mathrm{CH}_{3} \mathrm{OH}$ source-averaged column density from the hot component model to $\mathrm{H}_{2}$ source-averaged column density from the virial mass.

Column densities in the cold halo are over two orders of magnitude lower than in the hot core. The values given as lower limits for G13.87, G43.89, G45.12, G45.45 and G45.47 are good estimates of the true column densities as these cold halo components are optically thin and extended. All sources in which a hot $\mathrm{CH}_{3} \mathrm{OH}$ core was not seen have low halo column densities.

$\mathrm{CH}_{3} \mathrm{OH}$ fractional abundances for the hot cores in five out of seven sources are $310^{-8}$, from comparing the $\mathrm{CH}_{3} \mathrm{OH}$ source-averaged column density with the $\mathrm{H}_{2}$ source-averaged column density calculated from the virial mass. Exceptions are G34.26, which has twice this value, and G29.96, which has an extremely high abundance of $1.410^{-7}$ as a result of its low virial mass. The $\mathrm{CH}_{3} \mathrm{OH}$ abundance in the halo component ranges from $0.2-4$ $10^{-9}$, at most an order of magnitude less than in the cores.

$\mathrm{CH}_{3} \mathrm{OH}$ conditions in the $20^{\prime \prime}$ offset positions are similar to the conditions in the cold halo. Assuming temperatures of $20-30 \mathrm{~K}$, the column densities at the offset positions are reduced from the on-source column densities by a factor of between 2 and 10 .

\subsection{4. $\mathrm{HCOOCH}_{3}$}

Lines of $\mathrm{HCOOCH}_{3}$ (methyl formate) were observed in most of the line-rich sources (G9.62, G10.47, G29.96, G31.41 and G34.26). The detected lines range in excitation energies from $30-500 \mathrm{~K}$ and were all of A-type $\mathrm{HCOOCH}_{3}$, apart from one E-type line seen in G34.26. The observed lines were fairly weak $\left(T_{\mathrm{R}}^{*}<0.5 \mathrm{~K}\right)$ and broad $\left(\Delta v \sim 7 \mathrm{~km} \mathrm{~s}^{-1}\right)$. The lines generally occur in pairs which are close in frequency and usually blended, complicating the analysis somewhat. Rotation diagrams for methyl formate were attempted in three sources (G10.47, G31.41 and G34.26), but the $\mathrm{HCOOCH}_{3}$ data did not fit well to the rotation diagrams and no convincing results were obtained. No LTE fit was attempted because the lines are weak and blended. Lower limits to the beamaveraged column density were evaluated for most sources 
Table 7. $\mathrm{HCOOCH}_{3}$ column density upper and lower limits

\begin{tabular}{|c|c|}
\hline Source & $N_{\text {mol }}\left[10^{14} \mathrm{~cm}^{-2}\right]$ \\
\hline$\overline{\text { G9.62 }}$ & no unblended lines \\
\hline G10.30 & $<4.5$ \\
\hline G10.47 & $>16.0$ \\
\hline G12.21 & $<5.5$ \\
\hline G13.87 & $<4.4$ \\
\hline G29.96 & $>3.3$ \\
\hline G31.41 & $>10.0$ \\
\hline G34.26 & $>27.0$ \\
\hline G43.89 & $<4.5$ \\
\hline G45.12 & $<4.0$ \\
\hline G45.45 & $<4.0$ \\
\hline G45.47 & $<4.9$ \\
\hline G75.78 & $<4.5$ \\
\hline
\end{tabular}

where $\mathrm{HCOOCH}_{3}$ was detected. No such analysis was possible for G9.62, as there were no unblended lines observed. For G75.78, the line identifications were not secure, and we have calculated an upper limit for this and the remaining sources.

The large range of excitation energies possessed by the observed lines in each source suggests that it is highly likely that $\mathrm{HCOOCH}_{3}$ emission originates from regions of differing temperature and density and is thus not well characterised by the rotation diagram approach. The high excitation lines observed and the column density lower limits indicate that the emission must be from warm, fairly dense gas of beam-averaged column density $10^{14}-10^{16} \mathrm{~cm}^{-2}$. Again it is the most line-rich sources (G10.47, G34.26 and G31.41) that possess denser columns of methyl formate. The line-poor sources contain less than $\sim 10^{14} \mathrm{~cm}^{-2}$. This is in agreement, at least for G34.26, with other work by Macdonald et al. (1996) and Mehringer \& Snyder (1996) who have found methyl formate emission from G34.26 with temperature $150 \mathrm{~K}$ and column density $\sim 10^{16} \mathrm{~cm}^{-2}$.

\subsection{5. $\mathrm{C}^{18} \mathrm{O}$ and $\mathrm{C}^{17} \mathrm{O}$}

We observed four lines of $\mathrm{C}^{17} \mathrm{O}$ and $\mathrm{C}^{18} \mathrm{O}: \mathrm{C}^{18} \mathrm{O} 2-1$ $(219.560 \mathrm{GHz}), \mathrm{C}^{18} \mathrm{O} 3-2(329.331 \mathrm{GHz}), \mathrm{C}^{17} \mathrm{O} 2-1$ $(224.714 \mathrm{GHz})$ and $\mathrm{C}^{17} \mathrm{O} 3-2(337.061 \mathrm{GHz})$. Spectra were not taken of all these lines for all sources.

For sources where three or four CO lines were observed (G9.62, G29.96, G31.41, G34.26 and G75.78), column density, temperature and source size estimates for the COemitting region were made using a direct method of calculation, based on an assumption of LTE. Line parameters were measured by fitting Gaussians. From the ratio of the $\mathrm{C}^{18} \mathrm{O}$ to $\mathrm{C}^{17} \mathrm{O}$ lines, we estimated optical depth in each line (assuming the same excitation temperature in each species and a relative abundance of $\left[\mathrm{C}^{18} \mathrm{O}\right] /\left[\mathrm{C}^{17} \mathrm{O}\right]$ $=3.65$, Penzias 1981). Temperature and source size were calculated by comparing the line strengths of two transitions of a single species. For these five sources, the total source-averaged CO column density is calculated from each line strength using the temperature and source size estimates and assuming LTE. The results from each line are averaged to produce the quoted $N_{\mathrm{H}_{2}}$. Results from different lines are in agreement with each other to within $\pm 0.210^{24} \mathrm{~cm}^{-2}$

For the remaining sources, only one or two lines of CO were observed (G5.89, G10.30, G10.47, G12.21, G13.87, G43.89 and G45.47), and we could not determine optical depth, temperature and source size independently. For these sources, we calculate lower limits on the beamaveraged column density from the $\mathrm{C}^{17} \mathrm{O} J=3-2$ line.

Temperatures, source diameters and $\mathrm{H}_{2}$ column densities derived from $\mathrm{CO}$, assuming $X_{\mathrm{C}^{18} \mathrm{O}}=10^{-7}$, are given in Table 8.

The low temperatures $(20-40 \mathrm{~K})$ and large source sizes $\left(\sim 20^{\prime \prime}\right)$ confirm that $\mathrm{CO}$ is tracing material in the ambient cloud or halo region surrounding the compact cores. These low excitation lines are easily excited in cool gas, so the halo emission dominates over the beam-diluted core emission.

Hydrogen column densities from $\mathrm{C}^{18} \mathrm{O}$ and $\mathrm{C}^{17} \mathrm{O}$ are given in Table 8. Many of the sources have similar column densities, between $510^{23} \mathrm{~cm}^{-2}$ and $110^{24} \mathrm{~cm}^{-2}$. The lineweak sources (G10.30, G13.87, G43.89 and G45.47) have particularly low column densities. For G34.26, we estimate a high column density and high temperature. The hot core in this source has the largest angular size (from $\mathrm{CH}_{3} \mathrm{CN}$ and $\mathrm{CH}_{3} \mathrm{OH}$ results) and this may be influencing the results.

The mass of the halo can be estimated from $\mathrm{CO}$ in two ways: from the source-averaged $\mathrm{H}_{2}$ column density and using the virial theorem. Both estimates are given in Table 8, and range from a few hundred to a few thousand solar masses. Where we have only column density lower limits, we have estimated a virial mass assuming a source size of $0.5 \mathrm{pc}$ (the virial mass varies linearly with source size), and the masses from the column density are lower limits. The masses agree to within a factor of 8 , which is reasonable given the uncertainties in the quantities involved. Both $M_{\text {vir }}$ and $M_{\mathrm{CD}}$ from CO are remarkably consistent with the $\mathrm{C}^{34} \mathrm{~S}$ results of Cesaroni et al. (1991). Errors in the distance affect the mass estimates, as $M_{\mathrm{vir}} \propto d$ and $M_{\mathrm{CD}} \propto d^{2}$. However, in G9.62, G29.96, G31.41 and G34.26, the distances would have to be reduced by up to a factor of 5 , which is far in excess of any uncertainty in the distance estimates. Note that $M_{\mathrm{CD}}>M_{\mathrm{vir}}$ in four out of five sources for which both masses were determined. This suggests that the linewidth and therefore the virial mass is not being significantly increased by outflows or other systematic velocity variations. 
Table 8. CO results: temperature and source size; source-averaged $\mathrm{H}_{2}$ column density assuming $X_{\mathrm{C}^{18} \mathrm{O}}=10^{-7}$; linewidth, virial mass and mass derived from column density

\begin{tabular}{|c|c|c|c|c|c|c|}
\hline$\overline{\text { Object }}$ & $\begin{array}{l}T_{\mathrm{CO}} \\
{[\mathrm{K}]}\end{array}$ & $\begin{array}{l}D_{\mathrm{CO}} \\
{[\mathrm{pc}]}\end{array}$ & $\begin{array}{c}N_{\mathrm{H}_{2}} \\
{\left[\times 10^{24} \mathrm{~cm}^{-2}\right]}\end{array}$ & $\begin{array}{c}\Delta v \\
{\left[\mathrm{~km} \mathrm{~s}^{-1}\right]}\end{array}$ & $\begin{array}{c}M_{\text {vir }} \\
{\left[M_{\odot}\right]}\end{array}$ & $\begin{array}{l}M_{\mathrm{CD}} \\
{\left[M_{\odot}\right]}\end{array}$ \\
\hline 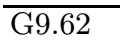 & 24 & 0.5 & 1.2 & 5.2 & 1400 & 4500 \\
\hline G29.96 & 33 & 0.7 & 0.7 & 3.4 & 800 & 5000 \\
\hline G31.41 & 24 & 0.7 & 0.7 & 5.5 & 2300 & 4600 \\
\hline G34.26 & 38 & 0.3 & 3.6 & 5.8 & 900 & 3700 \\
\hline G75.78 & 18 & 0.2 & 1.1 & 6.0 & 900 & 800 \\
\hline G5.89 & - & 0.5 & $>0.8$ & 6.8 & $2400^{*}$ & $>300$ \\
\hline G10.30 & - & $"$ & $>0.2$ & 5.2 & $1400^{*}$ & $>500$ \\
\hline G10.47 & - & $"$ & $>0.5$ & 7.8 & $3200^{*}$ & $>1200$ \\
\hline G12.21 & - & $"$ & $>0.3$ & 7.1 & $2600^{*}$ & $>3800$ \\
\hline G13.87 & - & $"$ & $>0.3$ & 2.7 & $400^{*}$ & $>400$ \\
\hline G43.89 & - & $"$ & $>0.1$ & 3.7 & $700^{*}$ & $>100$ \\
\hline G45.47 & - & $"$ & $>0.1$ & 3.8 & $800^{*}$ & $>200$ \\
\hline
\end{tabular}

* virial mass if a source size of $0.5 \mathrm{pc}$ is assumed.

Table 9. Column densities of sulphuretted species. Note that G45.12 and G45.45 have not been observed at any of the frequencies of the lines below and have been omitted from the table and that a dash denotes that the source has not been observed at the frequency of the line(s) in question

\begin{tabular}{lcccc} 
& \multicolumn{5}{c}{$N_{\text {mol }}\left[10^{12} \mathrm{~cm}^{-2}\right]$} \\
Source & $\mathrm{H}_{2} \mathrm{~S}$ & $\mathrm{SO}_{2}$ & $\mathrm{SO}$ & $\mathrm{C}^{34} \mathrm{~S}$ \\
\hline $\mathrm{G} 5.89$ & - & $>2300$ & $>7400$ & $>130$ \\
$\mathrm{G} 9.62$ & $>870$ & $>570$ & $>160$ & $>71$ \\
$\mathrm{G} 10.30$ & - & $<220$ & - & $<4.5$ \\
$\mathrm{G} 10.47>2200$ & $>1200$ & $280 \pm 190$ & $>47$ \\
$\mathrm{G} 12.21$ & - & $>320$ & $>120$ & $>21$ \\
$\mathrm{G} 29.96$ & $>710$ & $<250$ & $>110$ & $>35$ \\
$\mathrm{G} 31.41$ & $>840$ & $<330$ & $>150$ & $>41$ \\
$\mathrm{G} 34.26$ & $>2000$ & $>750$ & $>460$ & $>130$ \\
$\mathrm{G} 43.89$ & - & $<390$ & - & $>25$ \\
$\mathrm{G} 45.47$ & - & $<200$ & $>36$ & - \\
$\mathrm{G} 75.78$ & $>180$ & $470 \pm 90$ & $>250$ & $>160$ \\
\hline
\end{tabular}

\subsubsection{Sulphuretted species $\left(\mathrm{SO}_{2}, \mathrm{H}_{2} \mathrm{~S}, \mathrm{SO}\right.$ and $\left.\mathrm{C}^{34} \mathrm{~S}\right)$}

Sulphuretted molecules have been postulated to be a major chemical diagnostic of evolution in hot cores (Millar et al. 1997; Charnley 1997). We include results from all observed sulphur-bearing molecules in this section. The column densities derived from each molecule are summarised in Table 9.

Only one transition of $\mathrm{H}_{2} \mathrm{~S}$ was observed, the $2_{20}-2_{11}$ line at $216.71 \mathrm{GHz} . \mathrm{H}_{2} \mathrm{~S}$ was seen towards all sources observed at this frequency (G9.62, G10.47, G29.96, G31.41, G34.26 and G75.78). Lower limits to the column density have been evaluated and are summarised in Table 9 .

$\mathrm{SO}_{2}$ was detected towards 6 sources (G5.89, G9.62, G10.47, G12.21, G34.26 and G75.78), with excitation energies ranging from $100-500 \mathrm{~K}$. Sufficient lines for the rotation diagram analysis to be performed were seen towards G10.47, G34.26 and G75.78. Mainly moderate $(100-200 \mathrm{~K})$ excitation lines were observed towards G75.78 whereas a mixture of moderate and high excitation lines were observed toward G34.26 and G10.47. The G10.47 rotation diagram data do not fit well to a straight line and the temperature and beam-averaged column density of $\mathrm{SO}_{2}$ in G34.26 are poorly constrained. Too few lines were observed in each of these sources to construct separate high and low excitation rotation diagrams.

SO was seen towards most of our sources (G5.89, G9.62, G10.47, G12.21, G29.96, G31.41, G34.26, G45.47 and G75.78). The remaining sources (G10.30, G43.89, G45.12 and G45.45) were not observed at the frequencies of the detected SO lines. Only G10.47 possesses sufficient SO lines for a rotation diagram analysis, the temperature determined from this was $29 \pm 11 \mathrm{~K}$. Lower limits for the beam-averaged SO column density have been evaluated for the remaining sources and are summarised in Table 9.

The $\mathrm{C}^{34} \mathrm{~S} 7-6$ line at $337.06 \mathrm{GHz}$ was observed towards G5.89, G9.62, G10.47, G12.21, G29.96, G31.41, G34.26, G43.89 and G75.78. No $\mathrm{C}^{34} \mathrm{~S}$ was detected towards G10.30 and the remaining sources G45.12, G45.45, G45.47 and G13.87 were not observed at this frequency. $\mathrm{C}^{34} \mathrm{~S} 7-6$ has an excitation energy of $65 \mathrm{~K}$. Lower and upper limit column density analyses were performed and the results are given in Table 9 .

\subsubsection{Other molecules}

We discuss other important molecules detected in the survey $\left(\mathrm{C}_{2} \mathrm{H}_{5} \mathrm{CN}, \mathrm{CH}_{3} \mathrm{CHO}, \mathrm{CCH}\right.$ and $\left.\mathrm{HNCO}\right)$ in turn.

$\mathrm{C}_{2} \mathrm{H}_{5} \mathrm{CN}$ (ethyl cyanide) lines, with excitation energies of $130-290 \mathrm{~K}$, were seen towards G10.47, G31.41 and G34.26. The observed lines were fairly faint with $T_{\mathrm{R}}^{*}<$ $0.5 \mathrm{~K}$. It was found that the $\mathrm{C}_{2} \mathrm{H}_{5} \mathrm{CN}$ data from $\mathrm{G} 34.26$ did not fit to a rotation diagram. Single lines of $\mathrm{CH}_{2} \mathrm{CHCN}$ 
were detected towards G10.47 and G31.41, with a beamaveraged column density lower limit of $\sim 210^{14} \mathrm{~cm}^{-2}$ in both cases. This is in agreement with the column densities of $10^{14}-10^{15} \mathrm{~cm}^{-2}$ observed in G34.26 by Mehringer \& Snyder (1996).

$\mathrm{CH}_{3} \mathrm{CHO}$ (acetaldehyde) was tentatively detected in one or two transitions towards most sources in the survey (G9.62, G10.47, G12.21, G29.96, G31.41, G34.26 and G75.78). One transition was identified as the $7(3,4)-$ $7(2,6)$ at $236.52 \mathrm{GHz}$ and a second as $12(1,11)-11(1,10)$ $\mathrm{CH}_{3} \mathrm{CHO}$ at $235.99 \mathrm{GHz}$. Both transitions are of the Etype form of $\mathrm{CH}_{3} \mathrm{CHO}$. However, $\mathrm{CH}_{3} \mathrm{CHO}$ should have many other transitions in the observed bands which we did not detect, placing these identifications are in some doubt. The transition at $236.52 \mathrm{GHz}$ may be $\mathrm{HC}_{3} \mathrm{~N}$. Nevertheless, lower limit analyses were carried out for these sources; column densities are similar across all the sources at a few $10^{15} \mathrm{~cm}^{-2}$ with the exception of G10.47 which has a slightly higher beam-averaged column density lower limit of $1.410^{16} \mathrm{~cm}^{-2}$.

Two lines of $\mathrm{CCH}$ (the ethynl radical) were detected (the $J=9 / 2-7 / 2$ and $7 / 2-5 / 2$ doublets of the $N=4-3$ transition at $349 \mathrm{GHz}$ ) towards G9.62, G10.47, G12.21, G29.96, G31.41, G34.26, G45.47 and G75.78. The remaining sources were not observed at this frequency. The $\mathrm{CCH}$ lines are slightly blended with the $\mathrm{CH}_{3} \mathrm{CN} J=19-18$ $K=3 \& 4$ lines in most sources; in G34.26 the linewidths are such that the $\mathrm{CCH}$ lines are completely blended. Beam-averaged column density lower limits of $\sim 10^{14} \mathrm{~cm}^{-2}$ were evaluated for all sources.

HNCO (isocyanic acid) was detected towards G9.62, G10.30, G10.47, G29.96, G31.41, G34.26, G43.89 and G75.78. Most observed transitions of HNCO were unfortunately blended with each other or with methanol and could not be analysed. However lower limit analyses were possible for all the above sources except G10.30 and G43.89 and revealed that the beam-averaged column density of HNCO is fairly constant across all the sources at $\sim 10^{14} \mathrm{~cm}^{-2}$. HNCO was also detected at $20^{\prime \prime}$ offset positions in G9.62 and G34.26 with a beam-averaged column density lower limit of $\sim 510^{13} \mathrm{~cm}^{-2}$.

\section{Discussion}

In this section, we look at the survey as a whole, drawing together the results from individual molecules. We discuss the features that the cores have in common and the differences between them. We bring together what we have learned about physical conditions and structure from the various molecular tracers. Implications for our understanding of the environment for models of UC HII regions are discussed. The chemistry in hot cores is considered and we look at whether chemical models can predict the observed column densities.

\subsection{Physical conditions and structure}

The first striking point about this survey is that the number of lines detected varies greatly from source to source. In the $239 \mathrm{GHz}$ spectra, which cover 13 out of 14 sources, more than 30 molecular lines are detected in G10.47, G34.26 and G31.41 but only two low-excitation $\mathrm{CH}_{3} \mathrm{OH}$ lines are detected in G45.12, G45.45, 45.47, and 13.87. If the transitions observed in the survey are roughly divided into groups of low $(<50 \mathrm{~K})$ and high excitation energy, the low excitation lines are detected towards all sources whereas the high excitation lines are not. Examples of low excitation lines in this survey are $\mathrm{C}^{18} \mathrm{O}$ and $\mathrm{C}^{17} \mathrm{O}, \mathrm{C}^{34} \mathrm{~S}$ (though this was not detected towards G10.30) and the $\mathrm{CH}_{3} \mathrm{OH}$ lines at 241.767 and $241.797 \mathrm{GHz}$. High excitation lines include the $\mathrm{CH}_{3} \mathrm{CN} J=13-12$ lines, which were observed towards thirteen sources and detected towards eight of them $(J=19-18$ was detected in the fourteenth); $\mathrm{CH}_{3} \mathrm{OH}$ lines such as $J=12(1)-12(0) \pm$ at $336.865 \mathrm{GHz}$; and the $\mathrm{CH}_{3} \mathrm{CCH}$ lines in the $249 \mathrm{GHz}$ band.

High excitation energy lines require high temperatures to excite, so the non-detections mean that for a number of sources in this survey we have no evidence for hot cores. However, these sources have all previously been detected in $\mathrm{NH}_{3}(4,4)$ line which is $>200 \mathrm{~K}$ above ground, and therefore are known to have associated hot gas. The main reason for the non-detection of hot gas in the weakest emitters is simply limited sensitivity. The six line-poor sources in our survey are also the weakest $\mathrm{NH}_{3}(4,4)$ emitters (Cesaroni et al. 1991; Olmi et al. 1993), suggesting that the non-detections correspond to sources with a low column density of hot gas or small source size. The brightness of the high-excitation lines does not correlate with distance, pointing to intrinsic differences in the source properties, rather than simply beam-dilution effects.

Another factor which may have some effect on the line brightnesses is any offset between the pointing centre of the JCMT beam, which is $20^{\prime \prime}$ FWHM at $349 \mathrm{GHz}$, and the centre of the hot core. Such offsets could arise because of poor pointing or because the molecular cores are offset from the UC HII regions with which they are associated. However, an emission region with lines as bright as G34.26 or G10.47 would have to be offset by $\sim 10^{\prime \prime}$ or more from the beam centre in order to fail to be detected by this survey.

In the eight line-rich sources, the strength of high excitation energy lines of $\mathrm{CH}_{3} \mathrm{CN}, \mathrm{CH}_{3} \mathrm{OH}, \mathrm{CH}_{3} \mathrm{CCH}, \mathrm{SO}_{2}$ and other molecules indicate the existence of hot, dense gas. $\mathrm{CH}_{3} \mathrm{OH}$ and $\mathrm{CH}_{3} \mathrm{CN}$ are our best tracers of the physical conditions in the hot gas as we observed many emission lines of each spanning a wide range of excitation energies. From our LTE analyses of $\mathrm{CH}_{3} \mathrm{CN}$ and $\mathrm{CH}_{3} \mathrm{OH}$ in the five strongest sources (G9.62, G10.47, G29.96, G31.41 and G34.26), we found that the high excitation lines are emitted from a source or sources with a small beam filling 
factor. Both the detection of $\mathrm{CH}_{3}^{13} \mathrm{CN}$ and ${ }^{13} \mathrm{CH}_{3} \mathrm{OH}$ lines and the pattern of emission in $\mathrm{CH}_{3}^{12} \mathrm{CN}$ and ${ }^{12} \mathrm{CH}_{3} \mathrm{OH}$ are inconsistent with an extended, low column density source.

We have assumed for the purposes of our analysis that the small beam filling factor comes about because the emission comes from a single central condensation rather than widely scattered clumps. Interferometric observations of a few of the sources in the sample have shown single cores of $1-2^{\prime \prime}$ in hot $\mathrm{CH}_{3} \mathrm{CN}, \mathrm{NH}_{3}$ and other molecules (Cesaroni et al. 1994a,b; Olmi et al. 1996a,b; Mehringer \& Snyder 1996, Cesaroni et al. 1998) though in G9.62 multiple cores are resolved (Hofner et al. 1996). In some cores we have made observations $20^{\prime \prime}$ from the position of the UC HII region. These show little emission from hot gas: only in G9.62 and G34.26 do we see emission at the at $\left(0,20^{\prime \prime}\right)$ offset position in $\mathrm{CH}_{3} \mathrm{CCH}$, but not $\mathrm{CH}_{3} \mathrm{OH}$ or $\mathrm{CH}_{3} \mathrm{CN}$. If the cores were offset or scattered widely, we would expect to see hot gas in the $20^{\prime \prime}$ off positions. Our single-dish observations give no information on the shape of the core. Where necessary in the calculations we assume spherical symmetry.

In both $\mathrm{CH}_{3} \mathrm{CN}$ and $\mathrm{CH}_{3} \mathrm{OH}$, the hot cores are $0.02-$ $0.1 \mathrm{pc}$ in diameter $\left(1-5^{\prime \prime}\right.$ at the distance of G34.26). We see differences in the core size in different molecular tracers. The region of the hot core traced by $\mathrm{CH}_{3} \mathrm{OH}$ is larger than the $\mathrm{CH}_{3} \mathrm{CN}$, and there is some evidence that the lower energy transitions of $\mathrm{CH}_{3} \mathrm{CN}$ trace larger cores than the higher energy transitions. This is consistent with a model of hot cores in which there is a gradient of density and temperature increasing towards the centre, rather than a sharply defined boundary.

The physical conditions in the hot cores are extreme. On the $0.02-0.1 \mathrm{pc}$ scales traced by $\mathrm{CH}_{3} \mathrm{CN}$ and $\mathrm{CH}_{3} \mathrm{OH}$, densities are a few times $10^{7} \mathrm{~cm}^{-3}$ to $10^{8} \mathrm{~cm}^{-3}$, from virial masses. Even more extreme densities in G34.26, G10.47 and G31.41 are suggested by the observation of ${ }^{13} \mathrm{CH}_{3} \mathrm{OH}$, as the implied optically thick $\mathrm{CH}_{3} \mathrm{OH}$ component can be explained if densities reach $10^{9} \mathrm{~cm}^{-3}$. Where we have sufficiently accurate temperature determinations, we see that temperatures rise towards the centres of the cores: we see $\mathrm{CH}_{3} \mathrm{OH}$ with an average temperature of $50 \mathrm{~K}$; within that, the $\mathrm{CH}_{3} \mathrm{CN}$ region is $70 \mathrm{~K}$ or more; and within this region the excitation of vibrationally excited $\mathrm{CH}_{3} \mathrm{OH}$ suggests the presence of hot dust at temperatures of several hundred kelvin.

Strong emission in low excitation lines is visible in all sources. These low excitation lines account for all the line emission from the line-poor sources. From the temperatures $(\sim 20-30 \mathrm{~K})$ and source sizes $(\sim 0.5 \mathrm{pc})$ estimated from the analysis of $\mathrm{C}^{17} \mathrm{O}$ and $\mathrm{C}^{18} \mathrm{O}$, the low excitation emission is from cool gas that is more extended than the hot core. This "cold halo" component is also required for the $\mathrm{CH}_{3} \mathrm{OH}$ LTE model. Even in the cool gas, column densities in all the sources are high: as measured by $\mathrm{CO}, \mathrm{H}_{2}$ column densities range from $10^{23} \mathrm{~cm}^{-2}$ to $510^{24} \mathrm{~cm}^{-2}$. We note that high column densities were an indirect re- quirement of our selection procedure: these sources are all emitters of the $\mathrm{NH}_{3}(4,4)$ transition, and with typical ammonia fractional abundances of $10^{-7}$ column densities of $10^{23}-10^{24} \mathrm{~cm}^{-2}$ are required (Cesaroni et al. 1991).

The line-poor sources may have lower column densities in the halo than the line-rich sources. The cold halo $\mathrm{CH}_{3} \mathrm{OH}$ component varies in beam-averaged column density from less than $110^{14} \mathrm{~cm}^{-2}$ to $210^{15} \mathrm{~cm}^{-2}$, with upper limits only for the line-poor sources. This variation in $\mathrm{CH}_{3} \mathrm{OH}$ column density could be due to either underlying column density variation or to $\mathrm{CH}_{3} \mathrm{OH}$ abundance variation. However, the line-poor sources also lie at the low end of the $\mathrm{H}_{2}$ column density determinations from $\mathrm{CO}$, assuming (as we argue in the section on CO) that the column density lower limits are in fact fairly good estimates of the true column densities. Differences in halo column density could be linked with the non-detection of hot cores in some sources.

The line-poor sources appear to be missing the hot core component. In both $\mathrm{CH}_{3} \mathrm{CN}$ and $\mathrm{CH}_{3} \mathrm{OH}$, our excitation analysis shows that sources are less bright because they are smaller hot cores rather than because the source density is lower or because of temperature variations. Carried to its conclusion, this would suggest that the line-poor sources contain very small hot cores. We can put an upper limit on the $\mathrm{CH}_{3} \mathrm{OH}$ and $\mathrm{CH}_{3} \mathrm{CN}$ core sizes in the line-poor sources where we do not see any emission from hot gas. The smallest angular diameter core that we detect in $\mathrm{CH}_{3} \mathrm{OH}$ is G12.21 with a size of $1.3^{\prime \prime}$. The high excitation $\mathrm{CH}_{3} \mathrm{OH}$ lines in this source are 3 and 4- $\sigma$ detections. We would have detected $\mathrm{CH}_{3} \mathrm{OH}$ lines of half this strength, equivalent to a source size of $1^{\prime \prime}$. Similarly, a $\mathrm{CH}_{3} \mathrm{CN}$ hot core less than $0.8^{\prime \prime}$ would not have been detected. We observed $\mathrm{CH}_{3} \mathrm{CN} J=13-12$ in all sources in the survey, so we can rule out $\mathrm{CH}_{3} \mathrm{CN}$ hot cores bigger than $0.8^{\prime \prime}$ in the line-poor sources where this was not detected. Alternatively, the line-poor sources may contain hot gas of insufficient density to excite the high excitation transitions of $\mathrm{CH}_{3} \mathrm{CN}$ and $\mathrm{CH}_{3} \mathrm{OH}$ but yet be detectable in $\mathrm{NH}_{3} . \mathrm{CH}_{3} \mathrm{CCH}$ emission from hot gas is detected in more sources than $\mathrm{CH}_{3} \mathrm{CN}$ and $\mathrm{CH}_{3} \mathrm{OH}$ and could be tracing lower-density hot cores.

\subsection{Implications for UC HII models}

Wood \& Churchwell (1989) suggested that UC HiI regions must have lifetimes of $10^{5}$ years in order to explain their numbers. Various models have been proposed to explain the expansion of the UC regions in a way that reconciles their long lifetimes with their small sizes. Contenders include the bow shock model (Van Buren \& Mac Low 1992), champagne flow (Tenorio-Tagle et al. 1979), mass-loaded winds (Dyson et al. 1995; Redman et al. 1996; Williams et al. 1997; Lizano et al. 1996), modified Strömgen sphere models including neutral gas pressure (De Pree 
et al. 1995; Akeson \& Carlstrom 1996) and turbulence (Xie et al. 1996). These models all depend to some extent on the physical conditions in the immediate environment of the HiI region.

The two environmental parameters which we have measured that are most likely to affect the HII region expansion are density and linewidth (a measure of turbulence). We found no correlation of either of these parameters with size, when considering either hot core or halo tracers. In particular, we plotted our linewidths from $\mathrm{CH}_{3} \mathrm{CN}, \mathrm{CH}_{3} \mathrm{OH}, \mathrm{C}^{34} \mathrm{~S}$ and $\mathrm{C}^{17} \mathrm{O}$ and $\mathrm{C}^{18} \mathrm{O}$ against size to look for correlations, following Xie et al. (1996). Of the transitions chosen, the high excitation transitions of $\mathrm{CH}_{3} \mathrm{OH}$ at least fulfil Xie et al.'s conditions of high critical density and low optical depth, yet we found no correlation with UC Hir size. The hot core sizes do not correlate with the UC HiI sizes. We also looked for any evolution of shape or size of the HiI region in line with the chemical evolution of the core, without a positive result. In total, none of our measured environmental parameters correlates with UC HII region shape or size. The absence of correlation could be because we measure average conditions with a large beam rather than the conditions immediately surrounding the HiI region. Also both HII region and core evolve with time, and the conditions that we see now may not explain how the HII region has evolved in the past. A third option is that the HII region expansion depends on some environmental or other parameter that we have not measured in this study.

The cloud chemistry does provide a constraint on the Hir region ages. In our study of the chemical evolution of hot cores based on this survey, we calculate that the cold halos are less than $10^{5}$ years old (Hatchell et al. 1998, in preparation). Assuming that the UC HiI regions in our sample formed in the clouds in which they now lie, they must also be younger than $10^{5}$ years.

\subsection{Chemistry}

Hot cores have a rich and unique chemistry. Unlike cold dense clouds, hot cores have high densities of saturated molecules such as $\mathrm{NH}_{3}$ and $\mathrm{H}_{2} \mathrm{~S}$. These molecules are believed to form in icy mantles on dust grains, created from constituents deposited during the high density phases of collapse. As the cores heat up in the star formation process, the grain mantles are evaporated and the mantle species are released into the gas phase. A rich gas-phase chemistry proceeds in the hot gas, transforming many of the grain mantle products into daughter species on timescales of 100000 years.

We see high column densities of saturated molecules such as $\mathrm{H}_{2} \mathrm{~S}$ and $\mathrm{CH}_{3} \mathrm{OH}$. High abundances of saturated molecules are formed in grain ices as a result of rapid hydrogenation, so these observations confirm that grain mantle evaporation is important in hot cores (Millar

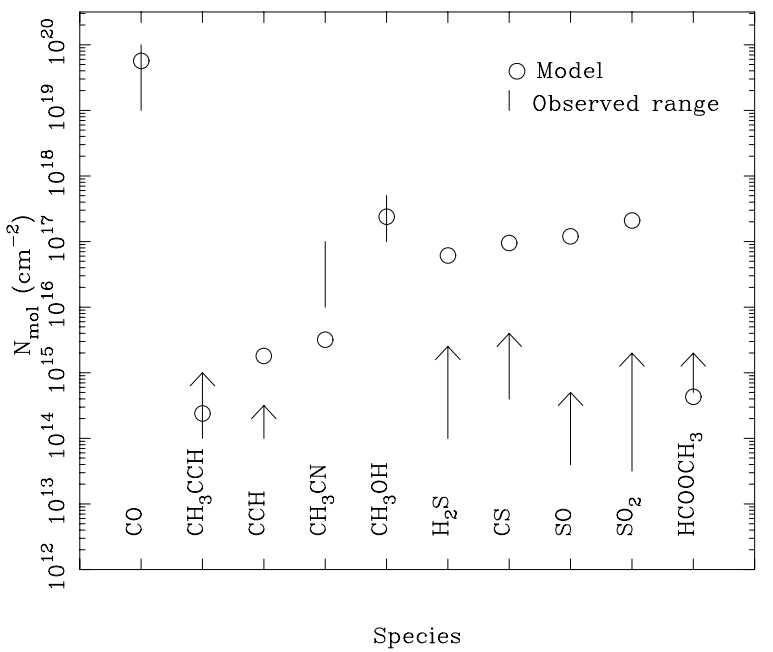

Fig. 5. Measured column densities compared to model predictions for column density through the core, from Millar et al. (1997) with a core age of $10^{4}$ years and a halo age of $10^{5}$ years. The model predictions are marked by circles. The range of column densities observed in the survey is shown by a line. Where the measured column densities are lower limits to the column density through the core this is shown by an arrow

et al. 1995; Mehringer \& Snyder 1996). Other direct or indirect products of grain surface chemistry that we observe in the hot cores are $\mathrm{CH}_{3} \mathrm{OCH}_{3}$ and $\mathrm{HCOOCH}_{3}$.

We have developed a model of hot core chemistry which follows the evolution of the chemistry in the core from the turn-on of core heating (Millar et al. 1997). The physical conditions used in this model were specifically tailored for G34.26 but can be generally applied to the other hot cores assuming they have similar temperatures and densities. In Fig. 5 we compare the column densities predicted by the model with those we have measured. The model predicts the total column density through the centre of the core, including contributions from core and halo, rather than the beam-averaged column density that we calculate for most molecules. The beam-averaged column density that we calculate is a lower limit on the column density through the core because of the effects of beam dilution on the core components.

Three species in particular are observed in larger amounts than the model predicts, as shown in Fig. 5. $\mathrm{CH}_{3} \mathrm{CN}$ column densities of $10^{16} \mathrm{~cm}^{-2}$ are marginally too high for this molecule to have formed in the gas phase, and if column densities are as large as $10^{17} \mathrm{~cm}^{-2}$ this suggests that there is also a surface chemistry route to its formation. $\mathrm{HCOOCH}_{3}$ column densities of $10^{16} \mathrm{~cm}^{-2}$ are larger than can be made in the gas in a hot core (Millar et al. 1997) and may indicate that grain surface reactions can produce this molecule, although the gas phase routes 
to $\mathrm{HCOOCH}_{3}$ are probably not yet clearly identified. The production of such a highly unsaturated molecule has not normally been considered to be a sign of grain chemistry and is at odds with the paradigm that surface reactions produce saturated molecules. This of course is only the case as long as there is sufficient atomic hydrogen to hydrogenate the surface radicals but it is possible that, in the final stages of grain accretion, the gas is depleted in atomic hydrogen so that saturation does not occur. In this context it is interesting to note that $\mathrm{HCOOCH}_{3}$ has been detected in Comet C/1995 O1 (Hale-Bopp) by Colom et al. (1997).

Although the model predicts enough $\mathrm{CH}_{3} \mathrm{CCH}$ to account for the column densities observed in some sources, in others the observed beam-averaged column density lower limit exceeds the amount of $\mathrm{CH}_{3} \mathrm{CCH}$ that can be produced by the model.

The chemistry of $\mathrm{CH}_{3} \mathrm{CCH}$ contained in Millar et al. (1997) is obviously inadequate in its description of $\mathrm{CH}_{3} \mathrm{CCH}$ in hot cores. A recently studied reaction which may be of importance in hot gas is that between $\mathrm{CH}$ and $\mathrm{C}_{2} \mathrm{H}_{4}$ (Canosa et al. 1997). Although the products have not been deterined as yet - the lowest energy products are $\mathrm{CH}_{3} \mathrm{CCH}$ and $\mathrm{CH}_{2} \mathrm{CCH}_{2}$, however - the reaction is fast with a rate coefficient of $\sim 410^{-10} \mathrm{~cm}^{3} \mathrm{~s}^{-1}$ over the temperature range $23-300 \mathrm{~K}$. If $\mathrm{CH}_{3} \mathrm{CCH}$ is the end product of this reaction, then methyl acetylene production can be enhanced in regions in which $\mathrm{C}_{2} \mathrm{H}_{4}$ is abundant, i.e. in the hot gas where it evaporates from grains. Using the above rate coefficient and abundances calculated by Millar et al. (1997) we find that production of methyl acetylene is enhanced most in the compact core of G34.3 where the $\mathrm{CH}+\mathrm{C}_{2} \mathrm{H}_{4}$ reaction is around a factor of ten larger than that due to $\mathrm{C}_{3} \mathrm{H}_{5}^{+}+\mathrm{e}$.

For most species in the survey for which we have column density estimates or limits $\left(\mathrm{CH}_{3} \mathrm{OH}, \mathrm{CO}, \mathrm{H}_{2} \mathrm{~S}, \mathrm{SO}_{2}\right.$, $\mathrm{SO}$, and $\mathrm{CS}$ ) the chemical model predicts column densities which are consistent with the data.

Because of the relatively rapid evolution of the chemistry, molecular abundances in hot cores can be used as chemical clocks to determine the onset of heating. We explore this possibility fully in a subsequent paper based on this survey (Hatchell et al. 1998, in preparation).

\section{Conclusions}

We have surveyed rotational transitions of molecular species towards 14 ultracompact HII regions, with the aims of observing the chemistry of molecular regions populated by species evaporated from grain mantles, for comparison with chemical models, looking in particular for any evidence of chemical evolution; and of determining the physical conditions of the molecular gas near a sample of UC HiI regions, to identify common characteristics, and for comparison with models of UC HII expansion. The main conclusions are as follows:
1. Eight out of fourteen sources - G5.89, G9.62, G10.47, G12.21, G29.96, G31.41, G34.26 and G75.78 - show high abundances of ice-evaporated species or their daughter products, confirming that grain mantle chemistry strongly influences the chemical composition of these star-forming regions. These sources are chemically rich and show many emission lines.

2. The eight line-rich sources show evidence for a corehalo structure, with a hot, dense core surrounded by cooler ambient cloud material. In five of these sources, there is evidence that the hot material is a central condensation rather than scattered clumps. There is a gradual increase of density and temperature towards the core, with higher excitation molecular tracers showing smaller cores. Densities in the cores reach at least $10^{8} \mathrm{~cm}^{-3}$ and temperatures are over $80 \mathrm{~K}$. Hot core sizes are of order $0.05 \mathrm{pc}$ (depending on the tracer) corresponding to $2^{\prime \prime}$ at $5 \mathrm{kpc}$. We did not find any evidence for hot cores in the line-poor sources G10.30, G13.87, G43.89, G45.12, G45.45, and G45.47. Any cores in these sources must be low density or small $\left(<1^{\prime \prime}\right)$.

3. We find no correlation between UC HII region size or shape and core size, chemical evolution, ambient cloud density, or linewidth.

4. Most of the measured column densities are satisfactorily predicted by our hot core chemical model, with the exception of $\mathrm{CH}_{3} \mathrm{CN}, \mathrm{HCOOCH}_{3}$, and $\mathrm{CH}_{3} \mathrm{CCH}$.

This survey has gone some way towards determining the nature of high mass star formation regions associated with UC HiI regions. Through observations of molecular lines we have measured the physical conditions and chemical conditions in 14 objects. The current generation of chemical models has proved quite successful in explaining many of the observed abundances in the hot cores and ambient clouds.

A number of outstanding questions remain about the structure and evolution of these regions. Are hot cores a necessary stage in the formation of high mass stars, or are they generated under special circumstances? What is the heating mechanism for hot cores? What are the interrelationships between hot cores, UC Hil regions and outflows? How good is our understanding of the chemistry?

Further observations and modelling are needed to answer these questions, including interferometric observations which show the spatial relationships between hot cores, UC HII regions and outflows, and single-dish observations to measure excitation and abundances. Future developments in modelling need to take account of the variation in physical conditions which accompanies the chemical evolution on similar timescales, and could potentially include details of the grain surface processes.

Acknowledgements. This research was supported by the UK Particle Physics and Astronomy Research Council (PPARC) through grants to UMIST and the University of Kent. JH and 
MAT are grateful to PPARC for funding their postdoctoral position and studentship, respectively. We would like to thank the JCMT staff for their support during the observations.

\section{Appendix A: Spectra}

We present full sets of spectra for each of the main frequency bands in the survey in Figs. 6-12. These figures are only available in the electronic version of the journal at http://www.edpsciences.com

\section{Appendix B: Line identifications and frequencies}

Tables 10-18 of line identifications for each source are available in electronic form at the CDS via anonymous ftp to cdsarc.u-strasbg.fr (130.79.128.5) or via http://cdsweb.u-strasbg.fr/Abstract.html

The tables give the measured parameters of observed frequency $\nu$ (obs), peak temperature $T_{\mathrm{R}}^{*}$ and linewidth $\Delta \nu_{1 / 2}$ for each line. Each independent detection of a particular line is given. The species, transition, rest frequency and upper level energy $\left(E_{\mathrm{u}} / k\right)$ are given for identified lines.

\section{References}

Acord J.M., Walmsley C.M., Churchwell E., 1997, ApJ 475, 693

Akeson R.L., Carlstrom J.E., 1996, ApJ 470, 528

Anderson T., De Lucia F.C., Herbst E., 1990a, ApJS 72, 797

Anderson T., Herbst E., De Lucia F.C., 1990b, ApJS 74, 647

Bachiller R., 1996, in E.F. van Dishoeck (eds.) Molecules in Astrophysics (IAU 178), p. 103

Blake G.A., Sutton E.C., Masson C.R., Phillips T.G., 1987, ApJ 315, 621

Boucher D., Burie J., Bauer A., Dubrulle A., Demaison J., 1980, J. Phys. Chem. Ref. Dat. 9, 3

Canosa A., Sims I.R., Travers D., Smith I.W.M., Rowe B.R., 1997, A\&A 323, 644

Cesaroni R., Hofner P., Walmsley C.M., Churchwell E., 1998, A\&A 331, 709

Cesaroni R., Walmsley C.M., Kömpe C., Churchwell E., 1991, A\&A 252, 278

Cesaroni R., Walmsley C.M., Churchwell E., 1992, A\&A 256, 618

Cesaroni R., Churchwell E., Hofner P., Walmsley C.M., Kurtz S., 1994a, A\&A 288, 903

Cesaroni R., Olmi L., Walmsley C.M., Churchwell E., Hofner P., 1994b, ApJ 435, L137

Charnley S.B., 1997, ApJ 481, 396

Churchwell E., Walmsley C.M., Cesaroni R., 1990, A\&AS 83, 119

Churchwell E., Walmsley C.M., Wood D.O.S., 1992, A\&A 253, 541

Codella C., Testi L., Cesaroni R., 1997, A\&A 325, 282

Colom P., Despois D., Germain B., et al., 1997, IAU Circ.
6645,1

De Pree C.G., Rodriguez L.F., Goss W.M., 1995, Rev. Mex. Astron. Astrofis. 31, 39-44

Dyson J.E., Williams R.J.R., Redman M.P., 1995, MNRAS 277,700

Gensheimer P.D., Mauersberger R., Wilson T.L., 1996, A\&A 314, 281

Goldsmith P.F., Krotkov R., Snell R.L., Brown R.D., Godfrey P., 1983, ApJ 274, 184

Hatchell J., Millar T.J., Thompson M.A., Macdonald G., 1998, A\&A (in press)

Hauschildt H., Güsten R., Phillips T.G., Schilke P., Serabyn E., Walker C.K., 1993, A\&A 273, L23

Helmich F.P., Jansen D.J., de Graauw Th., Groesbeck T.D., van Dishoeck E.F., 1994, A\&A 283, 626

Herbst E., 1996 (private communication)

Hofner P., Churchwell E., 1996, A\&AS 120, 283

Hofner P., Kurtz S., Churchwell E., Walmsley C.M., Cesaroni R., 1996, ApJ 460, 359

Hunter T.R., Phillips T.G., Menten K.M., 1997, ApJ 478, 283

Jacq T., Walmsley C.M., Henkel C., Baudry A., Mauersberger R., Jewell P.R., 1990, A\&A 228, 447

Lizano S., Canto J., Garay G., Hollenbach D., 1996, ApJ 468, 739

Lovas F.J., 1985, "Recommended rest frequencies for observed interstellar molecular microwave transitions", National Bureau of Standards (NBS), Washington

Lovas F.J., Suenram R.D., Snyder L.E., Hollis J.M., Lees R.M., 1982, ApJ 253, 149

Macdonald G.H., Gibb A.G., Habing R.J., Millar T.J., 1996, A\&AS 119, 333

Mehringer D.M., Snyder L.E., 1996, ApJ 471, 897

Millar T.J., Macdonald G.H., Gibb A.G., 1997, A\&A, 325, 1163

Millar T.J., Macdonald G.H., Habing R.J., 1995, MNRAS 273, 25

Olmi L., Cesaroni R., Walmsley C.M., 1993, A\&A 276, 489

Olmi L., Cesaroni R., Neri R., Walmsley C.M., 1996a, A\&A 315,565

Olmi L., Cesaroni R., Walmsley C.M., 1996b, A\&A 307, 599

Penzias A.A., 1981, ApJ 249, 518

Plume R., Jaffe D.J., Evans N.J., 1992, ApJS 78, 505

Poynter R.L., Pickett H.M., 1985, Appl. Opt. 24, 2235

Redman M.P., Williams R.J.R., Dyson J.E., 1996, MNRAS 280,661

Rodgers S.D., Millar T.J., 1996, MNRAS 280, 1046

Schultz A., Henkel C., Beckmann U., et al., 1995, A\&A 295, 183

Shepherd D.S., Churchwell E., 1996, ApJ 457, 267

Shepherd D.S., Churchwell E., Wilner D.J., 1997, ApJ 482, 355

Tenorio-Tagle G., 1977, A\&A 54, 517

Thompson M.A., Macdonald G.H., Millar T.J., 1998 (in preparation)

Turner B.E., 1991, ApJS 76, 617

Van Buren D., Mac Low M.-M., 1992, ApJ 394, 534

Williams R.J.R., Dyson J.E., Redman M.P., 1996, MNRAS 280,667

Wilner D.J., Ho P.T.P., Zhang Q., 1996, ApJ 462, 339

Wood D.O.S., Churchwell E., 1989, ApJS, 69, 831

Xie T., Mundy L.G., Vogel S.N., Hofner P., 1996, ApJ 473, 131 